\title{
FIRST ACETIC ACID SURVEY WITH CARMA IN HOT MOLECULAR CORES
}

\author{
Y.-S. JERRY SHIAO ${ }^{1,2}$, LESLIE W. LOONEY ${ }^{1}$, ANTHONY J. REMIJAN ${ }^{3,4}$, LEWIS E. \\ SNYDER $^{1}$, and DOUGLAS N. FRIEDEL ${ }^{1}$
}

\begin{abstract}
Acetic acid $\left(\mathrm{CH}_{3} \mathrm{COOH}\right)$ has been detected mainly in hot molecular cores where the distribution between oxygen $(\mathrm{O})$ and nitrogen $(\mathrm{N})$ containing molecular species is cospatial within the telescope beam. Previous work has presumed that similar cores with co-spatial $\mathrm{O}$ and $\mathrm{N}$ species may be an indicator for detecting acetic acid. However, does this presumption hold as higher spatial resolution observations become available of large $\mathrm{O}$ and N-containing molecules? As the number of detected acetic acid sources is still low, more observations are needed to support this postulate. In this paper, we report the first acetic acid survey conducted with the Combined Array for Research in Millimeterwave Astronomy (CARMA) at $3 \mathrm{~mm}$ wavelengths towards G19.61-0.23, G29.96-0.02 and IRAS 16293-2422. We have successfully detected $\mathrm{CH}_{3} \mathrm{COOH}$ via two transitions toward G19.61-0.23 and tentatively confirmed the detection toward IRAS 16293-2422 A. The determined column density of $\mathrm{CH}_{3} \mathrm{COOH}$ is $2.0(1.0) \times 10^{16} \mathrm{~cm}^{-2}$ and the abundance ratio of $\mathrm{CH}_{3} \mathrm{COOH}$ to methyl formate $\left(\mathrm{HCOOCH}_{3}\right)$ is $2.2(0.1) \times 10^{-1}$ toward G19.61-0.23. Toward IRAS $16293 \mathrm{~A}$, the determined column density of $\mathrm{CH}_{3} \mathrm{COOH}$ is $\sim 1.6 \times 10^{15} \mathrm{~cm}^{-2}$ and the abundance ratio of $\mathrm{CH}_{3} \mathrm{COOH}$ to methyl formate $\left(\mathrm{HCOOCH}_{3}\right)$ is $\sim 1.0 \times 10^{-1}$ both of which are consistent with abundance ratios determined toward other hot cores. Finally, we model all known line emission in our passband to determine physical conditions in the regions and introduce a new metric to better reveal weak spectral features that are blended with stronger lines or that may be near the 1-2 $\sigma$ detection limit.
\end{abstract}

Subject headings: ISM: abundances - ISM: individual (G19.61-0.23, G29.96-0.02, IRAS 16293-2422) — ISM: molecules — ISM: clouds

\footnotetext{
${ }^{1}$ Department of Astronomy, University of Illinois at Urbana-Champaign, Urbana, IL 61801, USA

${ }^{2}$ Physics Department, National Taiwan University, Taipei 10617, Taiwan; jshiao@phys.ntu.edu.tw

${ }^{3}$ National Radio Astronomy Observatory, Charlottesville, VA 2290, USA; aremijan@nrao.edu

${ }^{4}$ Center for Chemistry of the Universe, Department of Chemistry, University of Virginia, McCormack Rd., P.O. Box 400319, Charlottesville, VA 22904-4319
} 


\section{INTRODUCTION}

With high resolution observations, Sgr B2 has been resolved into many regions of compact molecular/continuum emission (Gaume et al. 1995); one of these regions, Sgr B2(N-LMH) (i.e., the "Large Molecule Heimat") (Snyder et al. 1994; Miao et al. 1995), is a molecule-rich hot core that has historically been the best place to search for large and complex molecules. Interstellar acetic acid $\left(\mathrm{CH}_{3} \mathrm{COOH}\right)$ was first detected in the hot molecular core (HMC) of Sgr B2(N-LMH) with the Berkeley-Illinois-Maryland Association (BIMA) and Owens Valley Radio Observatory (OVRO) millimeter-wavelength arrays by Mehringer et al. (1997). The compactness of the $\mathrm{CH}_{3} \mathrm{COOH}$ emission in Sgr B2(N-LMH) ( $\left.<3^{\prime \prime}\right)$ (e.g., Remijan et al. 2002) highlights the importance of interferometers. To clearly detect the compact emission features from $\mathrm{CH}_{3} \mathrm{COOH}$ and other large molecules toward hot cores embedded in regions of more extended molecular emission, high resolution interferometric observations are necessary. Thus, following the first $\mathrm{CH}_{3} \mathrm{COOH}$ detections in Sgr $\mathrm{B} 2(\mathrm{~N}-\mathrm{LMH})$ and using the BIMA array, Remijan et al. (2002) confirmed $\mathrm{CH}_{3} \mathrm{COOH}$ in Sgr B2(N$\mathrm{LMH}$ ) and discovered the second $\mathrm{CH}_{3} \mathrm{COOH}$ source, the hot core toward W51e2.

Remijan et al. (2003) launched an extensive $\mathrm{CH}_{3} \mathrm{COOH}$ survey of 12 galactic HMCs, including high-mass $\left(\geq 10 \mathrm{M}_{\odot}\right)$ and low-mass $\left(<10 \mathrm{M}_{\odot}\right)$ sources, and they found the third $\mathrm{CH}_{3} \mathrm{COOH}$ source toward the high-mass HMC G34.3+0.15. Using the IRAM 30-m telescope, Cazaux et al. (2003) reported the first detection of $\mathrm{CH}_{3} \mathrm{COOH}$ in IRAS $16293-2422$ via the $9_{*, 9}-8_{*, 8} E$ line at $100.855 \mathrm{GHz}$. This is the first low-mass star forming region with detected $\mathrm{CH}_{3} \mathrm{COOH}$ to date, making only a total of four known interstellar sources of $\mathrm{CH}_{3} \mathrm{COOH}$. At the conclusion of the search for new sources of $\mathrm{CH}_{3} \mathrm{COOH}$, it was found that the three high mass regions containing $\mathrm{CH}_{3} \mathrm{COOH}$ emission share certain chemical properties. For example, the abundances of $\mathrm{CH}_{3} \mathrm{COOH}$ and its isomer methyl formate $\left(\mathrm{HCOOCH}_{3}\right)$ are very different; in Sgr B2(N-LMH), the abundance of $\mathrm{HCOOCH}_{3}$ is 26 times higher than that of $\mathrm{CH}_{3} \mathrm{COOH}$ (Snyder 2006) assuming the emission from $\mathrm{CH}_{3} \mathrm{COOH}$ and $\mathrm{HCOOCH}_{3}$ are co-spatial within the telescope beam. In addition, it has been suggested by Remijan et al. (2004) that there is a relationship between the $\mathrm{CH}_{3} \mathrm{COOH}$ sources and the spatial separation of complex oxygen (O) (e.g. $\mathrm{HCOOCH}_{3}$ and $\left.\mathrm{CH}_{3} \mathrm{COCH}_{3}\right)$ and nitrogen $(\mathrm{N})$ containing species (e.g. $\mathrm{CH}_{3} \mathrm{CH}_{2} \mathrm{CN}$ and $\mathrm{NH}_{2} \mathrm{CH}_{2} \mathrm{CN}$ ). At the limit of the current observational resolution of these surveys, $\mathrm{CH}_{3} \mathrm{COOH}$ emission is only detected where the emission from $\mathrm{O}$ and $\mathrm{N}$-containing species is co-spatial. In contrast, there have not been any $\mathrm{CH}_{3} \mathrm{COOH}$ detections in HMCs with reported spatial separation of complex O and N-containing species, e.g. Orion KL (e.g. Sutton et al. 1995) and W3(OH) (e.g. Wyrowski et al. 1999), even though the observed separation is very dependent on source distance.

Gas phase and grain surface chemistry may both play critical roles in the formation of $\mathrm{CH}_{3} \mathrm{COOH}$

and other large molecules in the ISM (Hasegawa et al. 1992; Garrod \& Herbst 2006; Garrod et al. 2008). However, the exact pathways of $\mathrm{CH}_{3} \mathrm{COOH}$ formation in gas phase or on grain surfaces in astronomical environments are still not clear. The apparent spatial coincidence of the emission of complex $\mathrm{O}$ and $\mathrm{N}$-containing species with the $\mathrm{CH}_{3} \mathrm{COOH}$ emission may suggest a strong correlation with $\mathrm{CH}_{3} \mathrm{COOH}$ formation (e.g., Remijan et al. 2004). Another possibility is that $\mathrm{CH}_{3} \mathrm{COOH}$ and 
large N-bearing species share approximately the same time scale to form in hot cores. Nevertheless, it is important to investigate the spatial relationship between $\mathrm{CH}_{3} \mathrm{COOH}$ and the distribution of other complex molecular species. Thus, we also observed methyl formate $\left(\mathrm{HCOOCH}_{3}\right)$ and ethyl cyanide $\left(\mathrm{CH}_{3} \mathrm{CH}_{2} \mathrm{CN}\right)$ as proxies to determine the spatial distribution of $\mathrm{O}$ and $\mathrm{N}$-bearing species toward our sources in this survey.

To search for regions of $\mathrm{CH}_{3} \mathrm{COOH}$ emission and continue to determine its spatial relationship with the distribution of other complex molecular species, we have used the high sensitivity of the Combined Array for Research in Millimeter-wave Astronomy (CARMA) to carry out a $\mathrm{CH}_{3} \mathrm{COOH}$ survey toward three more hot cores. The observed sources are G19.61-0.23, G29.96-0.02 and IRAS 16293-2422 and are described in more detail in $\S 2$. A complete description of the CARMA observations are described in $\S 3$; the results are presented in $\S 4$; and in $\S 5$ we describe the analysis routine and discuss the implication of these observations with future surveys and chemical formation models.

\section{OBSERVED SOURCES}

\subsection{G19.61-0.23 \& G29.96-0.02}

G19.61-0.23 (hereafter, G19) is a molecule-rich ultracompact H II region, which includes a highmass HMC. Numerous molecules have been detected in this region, including OH (Matthews et al. 1977; Garav et al. 1985), $\mathrm{H}_{2} \mathrm{O}$ (Genzel \& Downes 1977), $\mathrm{CH}_{3} \mathrm{OH}$ (Kalenskii et al. 1994; Larionov et al. 1999), $\mathrm{NH}_{3}$ (Garav et al. 1998), CS (Shirlev et al. 2003; Wu \& Evans 2003; Larionov et al. 1999), HCN (Wu \& Evans 2003) and CO (Hofner et al. 2000). G29.96-0.02 (hereafter, G29) is another high-mass ultracompact region that also has been observed in many molecular species including $\mathrm{CH}_{3} \mathrm{CN}, \mathrm{C}^{18} \mathrm{O}, \mathrm{CH}_{3} \mathrm{OH}, \mathrm{CH}_{3} \mathrm{CH}_{2} \mathrm{CN}, \mathrm{C}^{34} \mathrm{~S}, \mathrm{CH}_{3} \mathrm{OCH}_{3}, \mathrm{HCOOCH}_{3},{ }^{34} \mathrm{SO}, \mathrm{SO}_{2}, \mathrm{HC}_{3} \mathrm{~N}, \mathrm{H}_{2} \mathrm{CS}$, $\mathrm{C}_{2} \mathrm{H}_{5} \mathrm{OH}$ and $\mathrm{SiO}$ (see e.g. Cesaroni et al. 1998; Olmi et al. 2003; Beuther et al. 2007, and references therein). One of the more recent surveys toward both sources was conducted by Fontani et al. (2007) with the Institute de Radioastronomie Millimétrique 30-m (IRAM) telescope. These observations targeted several emission features between 1 and $3 \mathrm{~mm}$ wavelengths of $\mathrm{CH}_{3} \mathrm{CH}_{2} \mathrm{CN}$, vinyl cyanide $\left(\mathrm{CH}_{2} \mathrm{CHCN}\right)$ and dimethyl ether $\left(\mathrm{CH}_{3} \mathrm{OCH}_{3}\right)$. Specifically, the observations by Fontani et al. (2007) were conducted to further investigate whether the so called "chemical differentiation" seen toward Orion and W3 is a general characteristic of high mass HMCs. However, no "chemical differentiation" was observed within the spatial limits defined by the size of the synthesized beam of their observations.

Toward G19, from the observations of a series of $\mathrm{CH}_{3} \mathrm{CH}_{2} \mathrm{CN}$ transitions, Fontani et al. (2007) determined a rotational temperature of $\mathrm{T}_{r o t}=116(12) \mathrm{K}$, and from that a source averaged column density of $N_{S}=2.2(0.3) \times 10^{16} \mathrm{~cm}^{-2}$. From a series of $\mathrm{CH}_{3} \mathrm{OCH}_{3}$ transitions that would be used to represent the excitation and distribution of other O-bearing molecules like $\mathrm{HCOOCH}_{3}$, Fontani et al. (2007) determined a $\mathrm{T}_{r o t}=158(17) \mathrm{K}$, and $N_{S}=2.0(0.2) \times 10^{15} \mathrm{~cm}^{-2}$. Note that the 
half power beam width (HPBW) of the IRAM 30-m telescope ranged in these $1 \mathrm{~mm}$ to $3 \mathrm{~mm}$ observations from $\sim 12^{\prime \prime}$ to $\sim 22^{\prime \prime}$, respectively. In contrast, using archival data from the Submillimeter Array (SMA), Wu et al. (2009) found a much higher temperature for the hot core regions. Using methyl cyanide $\left(\mathrm{CH}_{3} \mathrm{CN}\right)$ at a spatial resolution of 2 ' $6 \times 2$ '! 1 , they found $\mathrm{T}_{\text {rot }} \sim 552 \mathrm{~K}$ and the beam averaged column density, $N_{S} \sim 3.4 \times 10^{16} \mathrm{~cm}^{-2}$ without optical depth corrections.

In a dedicated $\mathrm{CH}_{3} \mathrm{COOH}$ survey of high mass HMCs by Remijan et al. (2004), $\mathrm{HCOOCH}_{3}$ (methyl formate), $\mathrm{CH}_{3} \mathrm{CH}_{2} \mathrm{CN}$ (ethyl cyanide), $\mathrm{CH}_{3} \mathrm{OH}$ (methanol) and $\mathrm{HCOOH}$ (formic acid) were all detected at a spatial resolution of $\sim 5^{\prime \prime}$ toward G19. There was also an indication of weak $\mathrm{CH}_{3} \mathrm{COOH}$ emission at $111.507 \mathrm{GHz}$, at the $1 \sigma$ detection limit of the BIMA array. Based on the detected $\mathrm{CH}_{3} \mathrm{COOH}$ sources and the upper limits from the non-detections, the abundance ratio of $\mathrm{CH}_{3} \mathrm{COOH}$ to $\mathrm{HCOOCH}_{3}$ seems universal in high-mass HMCs. If G19 indeed contains $\mathrm{CH}_{3} \mathrm{COOH}$, the $\mathrm{HCOOCH}_{3}$ detection indicated that the $\mathrm{CH}_{3} \mathrm{COOH}$ transitions were just slightly lower than the BIMA sensitivity. To clearly detect the $\mathrm{CH}_{3} \mathrm{COOH}$ transition lines, we needed a higher sensitivity by a factor of 4 , which is provided by CARMA in this survey.

Toward G29, the observations by Fontani et al. (2007) of $\mathrm{CH}_{3} \mathrm{CH}_{2} \mathrm{CN}$ transitions yielded a $\mathrm{T}_{\text {rot }}=121(17) \mathrm{K}$, and from that a $N_{S}=1.5(0.3) \times 10^{16} \mathrm{~cm}^{-2}$. Similarly, observations of $\mathrm{CH}_{3} \mathrm{OCH}_{3}$ transitions yielded a $\mathrm{T}_{\text {rot }}=141(26) \mathrm{K}$, and $N_{S}=6.2(1.0) \times 10^{15} \mathrm{~cm}^{-2}$. Another recent campaign toward G29 was by Beuther et al. (2007) who mapped the spatial distribution of several complex molecules with the SMA at a spatial resolution of $\sim 0^{\prime \prime} 4 \times 0^{\prime \prime} 3$. However, no temperature or column density determinations were made from the emission features of these complex molecules. The only reported temperature of the compact emission regions was made from observations of $\mathrm{CH}_{3} \mathrm{OH}$ in its vibrationally excited $v_{t}=1$ state. From the relative intensities of the detected emission features, Beuther et al. (2007) reported an excitation temperature of $\mathrm{T}_{e x} \sim 340 \mathrm{~K}$ with a lower limit of $220 \mathrm{~K}$. In addition, this mapping campaign showed the distributions of $\mathrm{CH}_{3} \mathrm{OH}, \mathrm{CH}_{3} \mathrm{CH}_{2} \mathrm{CN}, \mathrm{CH}_{3} \mathrm{OCH}_{3}$ and $\mathrm{HCOOCH}_{3}$ were co-spatial suggesting that $\mathrm{G} 29$ may be another source of $\mathrm{CH}_{3} \mathrm{COOH}$ and that an excitation temperature as high as $220 \mathrm{~K}$ may be used in the determination of the column density of these complex molecules (see $\S 3$ ).

\subsection{IRAS 16293-2422}

IRAS 16293-2422 is one of the most well-studied and observed low-mass star forming region (e.g., Looney et al. 2000). It consists of two clumps denoted as component A and B (hereafter I16293A and B). The structure of I16293A is much more complex than I16293B (e.g. Chandler et al. 2005). I16293A contains two centimeter sources A1 and A2 (Wootten 1989), and two submillimeter sources Aa and Ab (Chandler et al. 2005). Moreover, I16293A2 may actually be a bipolar ejection with two components (Loinard et al. 2007). Despite their low masses, both component A and B have been suggested to be as molecule-rich as high-mass HMCs (e.g. Schöier et al. 2002; Cazaux et al. 2003). 
Large O-bearing species, including $\mathrm{HCOOCH}_{3}$ and $\mathrm{HCOOH}$, and $\mathrm{N}$-bearing species, including $\mathrm{CH}_{3} \mathrm{CN}$ (methyl cyanide), $\mathrm{CH}_{2} \mathrm{CHCN}$ (vinyl cyanide) and $\mathrm{CH}_{3} \mathrm{CH}_{2} \mathrm{CN}$, have been detected toward this region (Cazaux et al. 2003; Bottinelli et al. 2004; Kuan et al. 2004; Remijan \& Hollis 2006). With the IRAM 30-m telescope, Cazaux et al. (2003) reported the first detection of $\mathrm{CH}_{3} \mathrm{COOH}$ in this low mass region via the $9_{*, 9}-8_{*, 8} E$ line at $100.855 \mathrm{GHz}$. This makes IRAS 16293-2422 the only low-mass $\mathrm{CH}_{3} \mathrm{COOH}$ source to date. However, Cazaux et al. (2003) did not detect the counterpart, $9_{*, 9}-8_{*, 8} A$ line, and the $9_{*, 9}-8_{*, 8} E$ line is known to be blended (e.g., Cazaux et al. 2003). Comparing the IRAM single dish of a $28^{\prime \prime}$ beam to an estimated source size of $5^{\prime \prime}$ for the $\mathrm{CH}_{3} \mathrm{COOH}$ emission, beam dilution would severely hinder the detection of other weak $\mathrm{CH}_{3} \mathrm{COOH}$ lines and may even pick up the extended emission from more distributed molecular species. To further investigate the $\mathrm{CH}_{3} \mathrm{COOH}$ distribution toward IRAS 16293-2422, we observed two more $\mathrm{CH}_{3} \mathrm{COOH}$ transitions in this survey with the high resolution of CARMA, which is better coupled to the assumed source size and is insensitive to extended emission.

\section{OBSERVATIONS}

The $\mathrm{CH}_{3} \mathrm{COOH}$ survey was carried out with the CARMA $\lambda=3 \mathrm{~mm}$ receivers from Spring 2006 to Spring 2008 in the B and C configurations. The individual angular resolutions are about $1^{\prime \prime}$ and $2^{\prime \prime}$, respectively. Table 1 summarizes the properties of the observed sources, which includes the source positions, flux calibrators, gain calibrators, distances, masses and LSR velocities. The six windows in the CARMA correlator were configured as two wide-band and four narrow-band windows. Each wide-band window has $500 \mathrm{MHz}$ bandwidth and 15 channels while each narrowband window has $31 \mathrm{MHz}$ bandwidth and 63 channels. The narrow-band high spectral resolution is $0.49 \mathrm{MHz}$ per channel, which provides sufficient sensitivity to resolve the observed transitions and lower the line confusion effect to detect the compact emission from large molecules. The amplitude and phase calibrations were accomplished by observing the gain and flux calibrators. The overall flux calibration uncertainty is $10-15 \%$. The CARMA flux uncertainty discussed in the text is the statistical uncertainty and does not account for any amplitude calibration error. In this paper, we will use the style of showing $1 \sigma$ statistical uncertainties in parenthesizes after the derived values. During data reduction, pointing and system temperatures were checked to monitor data quality.

We observed the $10_{*, 10}-9_{*, 9} E$ and $A \mathrm{CH}_{3} \mathrm{COOH}$ lines at $111.507270(20)$ and $111.548533(20)$ $\mathrm{GHz}$ (Ilvushin et al. 2008) in two narrow band windows. Based on previous observations (Remijan et al. 2002, 2003), they are unblended and have similar line strengths. In addition to $\mathrm{CH}_{3} \mathrm{COOH}$, we included two $\mathrm{HCOOCH}_{3}$ and three $\mathrm{CH}_{3} \mathrm{CH}_{2} \mathrm{CN}$ lines in the other narrow-band windows. Table 2 summarizes the observed molecules and their transitions. All spectral line data were taken from the Spectral Line Atlas of Interstellar Molecules (SLAIM) (F. J. Lovas, private communication)1, the Cologne Database for Molecular Spectroscopy (Müller et al. 2005) and the appropriate laboratory

\footnotetext{
${ }^{1}$ available at http://www.splatalogue.net (Remijan et al. 2007)
} 
references listed therein.

\section{RESULTS}

The observational data were reduced with the MIRIAD package (Sault et al. 1995). The final data presented in the figures were Hanning-smoothed over 3 channels and continuum-subtracted. The observations in $\mathrm{B}$ and $\mathrm{C}$ configurations were combined using natural weighting to obtain the best sensitivity and synthesized beams of $\sim 2^{\prime \prime}$. We present the observations of each source as both maps of the molecular emission and the spectra at the peak of the emission (Figures 1 through 7). The continuum emission maps shown were made from channels contained in the wideband windows which were free from line emission. All of the spectra from each source are shown in rows and columns. In each spectral figure, column (a) displays window 2 (rest frequency $=107.54375$ $\mathrm{GHz}$ ); column (b), window 3 (rest frequency=107.59404 GHz); column (c), window 6 (rest frequency $=111.50727 \mathrm{GHz}$ ); and column $(\mathrm{d})$, window 5 (rest frequency=111.54853 GHz). In row 1 , the observed spectra (Hanning smoothed over 3 channels) are overlaid on the modeled spectral line data represented by Gaussians (red trace). In row 2, the modeled spectral line data from row 1 are convolved with the spectral resolution of the original observational data and co-added with 24 $\mathrm{mJy} /$ beam random noise. Finally, row 3 presents the residuals from the modeled data subtracted from the observational data (i.e row 1 minus row 2). The maps and spectra are discussed below.

\subsection{G19.61-0.23}

One of the most relevant discoveries in this paper is the new detection of $\mathrm{CH}_{3} \mathrm{COOH}$ toward G19 that highlights the importance of interferometric observations in the detection of weak spectral signatures confined in compact emission regions. To illustrate the distribution of the $\mathrm{CH}_{3} \mathrm{COOH}$ emission toward G19, we have summed the intensity of the $\mathrm{CH}_{3} \mathrm{COOH}$ lines to produce a combined $\mathrm{CH}_{3} \mathrm{COOH}$ contour map which is overlaid on the continuum map in grayscale in Figure 1(a). Both the $10_{*, 10}-9_{*, 9} E$ and $10_{*, 10}-9_{*, 9} A$ lines were detected in the two $\mathrm{CH}_{3} \mathrm{COOH}$ spectral windows (Figures 2(c) and (d)) at the canonical $v_{L S R}$ of $40 \mathrm{~km} \mathrm{~s}^{-1}$ for G19. While some weak unidentified lines may appear in the windows after Hanning smoothing, the relative line strengths of the two $\mathrm{CH}_{3} \mathrm{COOH}$ transitions are roughly equal as predicted by rotational spectroscopy. G19 consists of several high density components of continuum emission between $2 \mathrm{~cm}$ and $3 \mathrm{~mm}$ wavelengths (Furuva et al. 2005). In this case, the $\mathrm{CH}_{3} \mathrm{COOH}$ emission is located near the center of component $\mathrm{C}$ and the $\mathrm{CH}_{3} \mathrm{COOH}$ emission region has an effective scale size of $2^{\prime \prime}$.

In addition to $\mathrm{CH}_{3} \mathrm{COOH}$, we have observed transitions of $\mathrm{HCOOCH}_{3}$ and $\mathrm{CH}_{3} \mathrm{CH}_{2} \mathrm{CN}$. Two transitions of $\mathrm{HCOOCH}_{3}, 9_{2,8}-8_{2,7} \mathrm{~A} / \mathrm{E}$ and three transitions of $\mathrm{CH}_{3} \mathrm{CH}_{2} \mathrm{CN}, 12_{4,9}-11_{4,8}, 12_{4,8}-$ $11_{4,7}$, and $12_{3,10}-11_{3,9}$ are shown in Figures 2 (a) and (b). All transitions are at a $v_{L S R}=40 \mathrm{~km}$

$\mathrm{s}^{-1}$. The statistical significance of three possible unidentified lines will be discussed in Section 
5.5. The $\mathrm{HCOOCH}_{3}$ map (Figure 1(b)) and $\mathrm{CH}_{3} \mathrm{CH}_{2} \mathrm{CN}$ map (Figure 1(c)) show emission that peaks primarily near component $\mathrm{C}$, like the $\mathrm{CH}_{3} \mathrm{COOH}$ emission, yet with a southward projection toward component A that was not seen in the lower resolution BIMA observations (Remijan et al. 2004). Table 3 summarizes the fitting results of the detected transitions towards G19. In our fitting routine, we assume that the rest frequencies of each molecular transition is fixed to the $v_{L S R}$ of the source and the $\mathrm{HCOOCH}_{3}$ transition that is blended with the $\mathrm{CH}_{3} \mathrm{CH}_{2} \mathrm{CN}$ transition shares the same linewidth and peak intensity as the unblended transition. Also, shown are the line widths and intensities of the model data for a direct comparison.

\subsection{G29.96-0.02}

We have detected $\mathrm{HCOOCH}_{3}$ and $\mathrm{CH}_{3} \mathrm{CH}_{2} \mathrm{CN}$ toward G29. Figures 3(a) and (b) show the distribution of $\mathrm{HCOOCH}_{3}$ and $\mathrm{CH}_{3} \mathrm{CH}_{2} \mathrm{CN}$ emission toward the $\mathrm{G} 29$ region, respectively. In Figure 4. we clearly detected the two blended pairs of transitions of $\mathrm{HCOOCH}_{3} 9_{2,8}-8_{2,7} E / A$ and $\mathrm{CH}_{3} \mathrm{CH}_{2} \mathrm{CN} 12_{4,9}-11_{4,8}$ and $12_{4,8}-11_{4,7}$. The spectrum is very similar to that of G19 (Figure 2(a)). The $\mathrm{CH}_{3} \mathrm{CH}_{2} \mathrm{CN} 12_{3,10}-11_{3,9}$ transition and possible $\mathrm{U}$ lines in Figure 4 (b), also have similar profiles to those in Figure 2(b).

G29 consists of two main continuum peaks at $1.2 \mathrm{~cm}$, which trace the UCH II region, and at

$862 \mu \mathrm{m}$, which trace the cloud core (Beuther et al. 2007). The cloud core is the location of the molecular emission; however, the emission is not bright enough to contribute significantly at $3 \mathrm{~mm}$ wavelengths. In Figure 3 the continuum emission is dominated by the UC H II region.

Figure 4(c) and (d) shows the two $\mathrm{CH}_{3} \mathrm{COOH}$ spectral windows toward G29. Unlike the data from $\mathrm{G} 19$, there are no statistically significant features at the $\mathrm{CH}_{3} \mathrm{COOH}$ rest frequencies. There is a $1 \sigma$ feature that may be due to the $10_{*, 10}-9_{*, 9} E$ transition of $\mathrm{CH}_{3} \mathrm{COOH}$, but the corresponding $10_{*, 10}-9_{*, 9} A$ transition is noticeably absent. In the discussion, we will investigate these passbands more closely with respect to the modeled data. Table 4 summarizes the fitting results of the detected transitions towards G29. The fitting criteria and model predictions are presented in the same manner as in Table 3 ,

\subsection{IRAS 16293-2422}

Unlike the spectra from Cazaux et al. (2003), we resolved IRAS 16293-2422 into I16293A and $\mathrm{B}$ with our $3^{\prime \prime} .1 \times 1^{\prime \prime} .9$ beam (see Figure 5). Therefore, beam dilution in this observation is minimized. Figure 6 and 7 shows the two $\mathrm{CH}_{3} \mathrm{COOH}$ spectral windows toward I16293A and I16293B, respectively. Toward I16293A, we detect weak emission features that may be due to transitions of $\mathrm{CH}_{3} \mathrm{COOH}$ but they are right at the $2 \sigma$ detection limit. Toward I16293B, as in G29, there are no statistically significant features at the $\mathrm{CH}_{3} \mathrm{COOH}$ rest frequencies. There are $1 \sigma$ features that may be due to the $10_{*, 10}-9_{*, 9} A / E$ transitions in these Hanning smoothed data, but 
nothing convincing beyond that level. In $\S 5.5$ we evaluate whether the features toward I16293A may be due to $\mathrm{CH}_{3} \mathrm{COOH}$.

We have also observed two new transitions of $\mathrm{HCOOCH}_{3}$. The line profiles of $\mathrm{HCOOCH}_{3}$ shown in Figure 6 toward I16293A are wider and may be suffering from self absorption (Remijan \& Hollis 2006) more than the line profiles shown in Figure 7 toward I16293B, which are much more narrow. This suggests that I16293A and I16293B may have distinguishable molecular dynamics. Figure 5 (a) and (b) show the distribution of $\mathrm{HCOOCH}_{3}$ emission with respect to the $3 \mathrm{~mm}$ continuum emission toward the I16293A \& B regions, respectively. Table 5 summarizes the fitting results of the detected transitions towards IRAS 16293-2422. The fitting criteria and model predictions are presented in the same manner as in Table 3 ,

\section{ANALYSIS \& DISCUSSION}

\subsection{Molecular Column Densities}

Molecular column density is one essential physical parameter needed to constrain chemical models (see e.g. Garrod et al. 2008 and references therein). In order to determine the column densities of each molecular species of interest, we assume that each region has uniform physical conditions, that the populations of the energy levels can be characterized by a Boltzmann distribution, and finally, that the emission is optically thin. Assuming that the molecular species is in local thermodynamic equilibrium (LTE) and low optical depth, the total beam averaged column density is

$$
N_{T}\left(\mathrm{~cm}^{-2}\right)=2.04 \times 10^{20} \frac{\int I\left(\mathrm{Jy} \mathrm{beam}^{-1}\right) d v\left(\mathrm{~km} \mathrm{~s}^{-1}\right) Q_{r} e^{E_{u}(K) / T_{r}}}{\Omega_{b}\left(\operatorname{arcsec}^{2}\right) \nu^{3}\left(\mathrm{GHz}^{3}\right)<S \mu^{2}>\left(\text { debye }^{2}\right)},
$$

where $\Omega_{b}$ is the solid angle of the beam , $\int I_{v} d v$ is the integral of the line intensity over velocity, $\nu$ is the spectral line frequency, $\left\langle S \mu^{2}\right\rangle$ is the line strength parameter, $Q_{r}$ is the rotational partition function, $T_{r}$ is the rotational temperature, and $E_{u}$ is the upper level energy of the transition (Miao et al. 1995). In each case, we assume the source emission fills the synthesized beam of the observations. All of the line parameters used for the analysis are given in Table 2.

A least-square fitting routine with Gaussian functions was used to determine the linewidths $(\Delta v)$, peak intensities $(\Delta \mathrm{I})$ and $\int I d v$ of each observed spectral line toward each source. All least-square fitting was done using the standard packages contained in the Mathematica software package. Using the spectroscopic parameters from Table 2 and by varying the temperature and column density over a wide range of values (e.g. the temperature range investigated was from $10-500 \mathrm{~K}$ and the column density range was from $10^{12}-10^{18} \mathrm{~cm}^{-2}$ for each molecular species), Equation (1) gave a predicted integrated line intensity for each spectral line observed in our data. These predictions of integrated line intensity were then compared to the measured values and, using a least-squares fitting routine, a best fit temperature and column density was found for each

molecule toward each source. However, there were some sources where an independent fit of the 
temperature did not converge. These sources are described in more detail in the following sections. Columns 3 and 4 of Table 3, 4 and 5 give the fitted line parameters of the observed peak intensity and line width. Columns 5 and 6 give the modeled peak intensity based on the least square fitting routine and the fitted line width from the observed data, respectively. Once all the relevant line parameters have been determined, a model spectrum with the appropriate amount of random noise added and convolved with the spectral resolution of the observation can then be produced and be directly compared with the observed dataset (see $\S 5.5$ ).

The rotational temperature $T_{r}$ generally is representative of the physical kinetic temperature in regions where the spatial density is larger than the critical density of the molecule in question. The assumption in this work is that the densities of the HMCs investigated are expected to be high enough for this to be true for these molecules. The rotational temperature is often found by observing several different transitions of a molecular species over a range of $E_{u}$ values. However, given the limited bandwidth and low number of transitions observed in our data, it was often difficult to accurately constrain the temperature and column density of our three observed molecules toward these regions (e.g., Snyder et al. 2005). Therefore, when the least squares fit failed to give a reasonable temperature for a given molecular species, we adopted a rotational temperature from previous observations by other investigators toward these sources at similar spatial resolution and were then able to fit for the total beam averaged column densities or associated upper limits if no transitions were detected beyond the statistically significant $1 \sigma$ limit. It was also the case that if the fitting procedure was given too many free parameters, a constraint was needed on one or more or else it would not converge (see the G29 discussion below). To properly constrain the temperature of these regions over a variety of molecular species, observations of additional transitions are necessary and complete spectral line surveys may be required (see e.g. Fontani et al. 2007; Friedel et al. 2004, and references therein).

For the G19 region, the least square fitting routine determined a rotational temperature of $\mathrm{CH}_{3} \mathrm{CH}_{2} \mathrm{CN}$ of $\mathrm{T}_{\text {rot }}=161(58) \mathrm{K}$, and a beam averaged column density of $N_{T}=6(3) \times 10^{16} \mathrm{~cm}^{-2}$. The errors on all fits are $1 \sigma$. The routine could not find a reasonable fit for the temperature and column density for either $\mathrm{HCOOCH}_{3}$ or $\mathrm{CH}_{3} \mathrm{COOH}$ presumably because the detected transitions were too close in energy. Therefore, because the emission regions of each molecular species around G19 are nearly co-spatial (Figure 1), we adopted the same rotational temperature for $\mathrm{HCOOCH}_{3}$ and $\mathrm{CH}_{3} \mathrm{COOH}$ as found from the $\mathrm{CH}_{3} \mathrm{CH}_{2} \mathrm{CN}$ fit. Assuming a $\mathrm{T}_{\text {rot }}=161 \mathrm{~K}$ for $\mathrm{HCOOCH}_{3}$, we find a best fit to the column density of $N_{T}=9(2) \times 10^{16} \mathrm{~cm}^{-2}$ and for $\mathrm{CH}_{3} \mathrm{COOH}$, we find a best fit to the column density of $N_{T}=2(1) \times 10^{16} \mathrm{~cm}^{-2}$. Given the minimum and maximum values of the measured column density, we determine an abundance ratio range of $\mathrm{N}_{\mathrm{HCOOCH}_{3}} / \mathrm{N}_{\mathrm{CH}_{3} \mathrm{COOH}}=3-11$. Table 3 gives the fitted intensities of each of the detected spectral features compared to the predicted intensities determined from Equation (1) for G19.

For the G29 region, the least square fitting routine determined a rotational temperature of $\mathrm{CH}_{3} \mathrm{CH}_{2} \mathrm{CN}$ of $\mathrm{T}_{\text {rot }}=107(49) \mathrm{K}$, and a beam averaged column density of $N_{T}=1.1(7) \times 10^{16} \mathrm{~cm}^{-2}$ only if we adopted a rotational temperature of $150 \mathrm{~K}$, determined from Olmi et al. (2003) and 
supported by the observations of Fontani et al. (2007) and Beuther et al. (2007), for the transitions of $\mathrm{HCOOCH}_{3}$. This temperature was also set to determine the upper limit for the column density of $\mathrm{CH}_{3} \mathrm{COOH}$. Assuming a $\mathrm{T}_{\text {rot }}=150 \mathrm{~K}$ for $\mathrm{HCOOCH}_{3}$, we find a best fit to the column density of $N_{T}=4(1) \times 10^{16} \mathrm{~cm}^{-2}$ and for $\mathrm{CH}_{3} \mathrm{COOH}$, we find an upper limit to the column density of $N_{T}<9 \times 10^{14} \mathrm{~cm}^{-2}$ for a relative abundance ratio upper limit of $\mathrm{N}_{\mathrm{HCOOCH}} / \mathrm{N}_{\mathrm{CH}_{3} \mathrm{COOH}}>50$. Table 4 gives the fitted intensities of each of the detected spectral features compared to the predicted intensities determined from equation (1) for G29. We also note the detection of another feature shown in Figure 2 near $107.540 \mathrm{GHz}$ that is most likely due to two high energy (>110 K) spectral lines of $\mathrm{CH}_{3} \mathrm{CH}_{2} \mathrm{CN}$. The discussion of weak spectral features near the $1 \sigma$ noise level will be given in section $\S 5.5$.

A recent observation of the G19 and G29 regions with the IRAM 30-m telescope (Fontani et al. 2007) covered our two acetic acid line frequencies (Figure 8). However, with a $28^{\prime \prime}$ beam, the lines detected are under the noise level of $26 \mathrm{mK}$. These lines are brighter than those observed by Cazaux et al. (2003), but the beam dilution is severe. The high resolution capability of CARMA is indeed critical for searching for acetic acid and large molecule research.

Finally, toward IRAS 16293-2422, the least square fitting routine could not determine a rotational temperature for the detected transitions of $\mathrm{HCOOCH}_{3}$ presumably for the same reason as described toward G19. Thus, we adopted a rotational temperature of $62 \mathrm{~K}$ determined from Bottinelli et al. (2004). In this case, we find a best fit to the column density of $\mathrm{HCOOCH}_{3}$ of $N_{T}=1.5(3) \times 10^{16} \mathrm{~cm}^{-2}$ toward region A and $6(1) \times 10^{15} \mathrm{~cm}^{-2}$ toward region B. Since there was no clear detection of $\mathrm{CH}_{3} \mathrm{CH}_{2} \mathrm{CN}$ beyond the $1 \sigma$ detection limit toward either region $\mathrm{A}$ or $\mathrm{B}$, based on the noise level in each of the passbands containing those lines, we determined an upper limit to the total beam averaged column density of $\mathrm{CH}_{3} \mathrm{CH}_{2} \mathrm{CN}$ to be $<6 \times 10^{13} \mathrm{~cm}^{-2}$ for region $\mathrm{A}$; and $<3 \times 10^{14} \mathrm{~cm}^{-2}$ toward region $\mathrm{B}$. For $\mathrm{CH}_{3} \mathrm{COOH}$, the $2 \sigma$ detection toward region A gives a total beam averaged column density of $\sim 1.6 \times 10^{15} \mathrm{~cm}^{-2}$ and for region $\mathrm{B}$, we determine and upper limit of $<6 \times 10^{14} \mathrm{~cm}^{-2}$. Table 5 summarizes the fitting results of the $\mathrm{HCOOCH}_{3}$ transitions compared to the model intensities determined from Equation (1).

\subsection{A Proxy for the Detection of $\mathrm{CH}_{3} \mathrm{COOH}$ Sources}

We have successfully detected $\mathrm{CH}_{3} \mathrm{COOH}$ toward G19. Following Sgr B2(N-LMH), W51e2 and G34.3+0.15, G19.61-0.23 is the fourth high mass hot core that is a source of $\mathrm{CH}_{3} \mathrm{COOH}$. We compare the $\mathrm{CH}_{3} \mathrm{COOH}$ column densities and the abundance ratios to $\mathrm{HCOOCH}_{3}$ of all detected $\mathrm{CH}_{3} \mathrm{COOH}$ hot cores in Table 7 . The values are generally consistent. Therefore, the column densities and ratios can be used to constrain hot core chemical models assuming the co-spatial dependence of $\mathrm{HCOOCH}_{3}$ and $\mathrm{CH}_{3} \mathrm{COOH}$ remains. This has yet to be tested at extremely high spatial resolution. In addition, a consistency can be found between all the sources containing $\mathrm{CH}_{3} \mathrm{COOH}$ emission by comparing the relative line strengths between the detected transitions of $\mathrm{HCOOCH}_{3}$ and $\mathrm{CH}_{3} \mathrm{CH}_{2} \mathrm{CN}$ to the $\mathrm{CH}_{3} \mathrm{COOH}$ emission. Taking a ratio between measured (or predicted inten- 
sities) of the $107.594 \mathrm{GHz}$ line of $\mathrm{CH}_{3} \mathrm{CH}_{2} \mathrm{CN}$ to the $111.507 \mathrm{GHz}$ line of $\mathrm{CH}_{3} \mathrm{COOH}$, we find that this ratio is between 5.5 and 6 for those sources where $\mathrm{CH}_{3} \mathrm{COOH}$ is detected. Therefore, given the line intensity of $0.21 \mathrm{Jy} /$ beam for the $107.594 \mathrm{GHz}$ line of $\mathrm{CH}_{3} \mathrm{CH}_{2} \mathrm{CN}$ toward $\mathrm{G} 29.96-0.02$, the expected line intensity for the $111.507 \mathrm{GHz}$ line of $\mathrm{CH}_{3} \mathrm{COOH}$ is $\leq 0.04 \mathrm{Jy} /$ beam. This intensity is right at the $1 \sigma$ detection limit of the current CARMA observations. Therefore, in addition to the criteria for the detection of $\mathrm{CH}_{3} \mathrm{COOH}$ outlined in Remijan et al. (2003), we now have a good proxy for the detectability of the $111 \mathrm{GHz} \mathrm{CH}_{3} \mathrm{COOH}$ transitions relative to the detected line strengths of the $\mathrm{HCOOCH}_{3}$ and $\mathrm{CH}_{3} \mathrm{CH}_{2} \mathrm{CN}$ transitions near $107 \mathrm{GHz}$. Further observations of new sources of $\mathrm{HCOOCH}_{3}$ and $\mathrm{CH}_{3} \mathrm{CH}_{2} \mathrm{CN}$ that fit the criteria set by Remijan et al. (2003) and higher S/N observations of sources like G29, are necessary to validate this hypothesis.

\section{3. $\mathrm{CH}_{3} \mathrm{COOH}$ in IRAS 16293-2422?}

High spatial resolution maps and spectra of IRAS 16293-2422 clearly reveal that the morphologies of the emission of complex molecules are clearly not co-spatial and that there is no simple separation of $\mathrm{O}$ and N-bearing "chemical differentiation" (Guélin et al. 2008). Blake et al. (1987) and Rodgers \& Charnley (2001) have suggested the difference in the morphologies of the emission of complex molecules in these regions reflects the timescales of chemical evolution. Predominantly N-bearing species (e.g. $\mathrm{CH}_{3} \mathrm{CH}_{2} \mathrm{CN}$ ) represent the later stage of chemical evolution while predominantly O-bearing species (e.g. $\mathrm{HCOOCH}_{3}$ ) represent the earlier stage. Their models can estimate the ages of hot cores by comparing this difference between the $\mathrm{O}$ and $\mathrm{N}$ morphology of emission. However, to date, no direct observation can accurately measure hot core ages, so the uncertainty of these estimates is large.

IRAS 16293-2422 also shows clear distinctions in the morphology of molecular emission (Chandler et al. 2005; Remijan \& Hollis 2006). In fact, the relative distributions of molecular species will undoubtedly give insight into their formation chemistry. It may also turn out that the relative abundances of molecular species and the overall population of energy levels of the detected transitions of a given molecule may be determined more by the formation chemistry and not on the local thermodynamic environment. To test this hypothesis will require high spectral and spatial resolution observations of a group of species that may form via a common formation pathway.

IRAS 16293-2422 has been reported as the first low-mass $\mathrm{CH}_{3} \mathrm{COOH}$ source by Cazaux et al. (2003) using the IRAM 30-m telescope, but the one detected line was likely blended. While IRAS 16293-2422 is not resolved in their work (beamsize of $28^{\prime \prime}$ ), we have resolved it into I16293A and $\mathrm{B}$ with a separation of less than $5^{\prime \prime}$. Even though their observation used different transitions, we should detect our $\mathrm{CH}_{3} \mathrm{COOH}$ lines in our synthesized beam of 3 "' $1 \times 1$ 1".9 using their flux estimates. Although at a lower flux than expected from their results, we do detect two $\mathrm{CH}_{3} \mathrm{COOH}$ transitions toward region A but only at the $2 \sigma$ limit. We estimate a column density of $\sim 1.6 \times 10^{15} \mathrm{~cm}^{-2}$, and Cazaux et al. (2003) estimated an upper limit of $2.5 \times 10^{15} \mathrm{~cm}^{-2}$, which is similar. 
Furthermore, there is no evidence beyond the $1 \sigma$ levels of any emission of $\mathrm{CH}_{3} \mathrm{COOH}$ toward region $\mathrm{B}$. In addition, while we have observed $\mathrm{HCOOCH}_{3}$ in $\mathrm{I} 16293 \mathrm{~A}$ and $\mathrm{B}$, we did not detect the corresponding $\mathrm{CH}_{3} \mathrm{CH}_{2} \mathrm{CN}$ emission features. The detection of $\mathrm{CH}_{3} \mathrm{COOH}$ toward $\mathrm{G} 19$ (and possibly toward I16293A) and the other hot cores have demonstrated that a diversity of abundant large $\mathrm{O}$ and $\mathrm{N}$-bearing species are strongly correlated with $\mathrm{CH}_{3} \mathrm{COOH}$. One possibility is that the mix of $\mathrm{O}$ and N-bearing species may serve as an efficient chemical network for $\mathrm{CH}_{3} \mathrm{COOH}$ and other large molecules. Thus, they need to wait for large N-bearing molecules to form first before they can be efficiently produced. Another possibility is that the formation of large $\mathrm{O}$ and N-bearing species is simply time dependent, and the gas-phase $\mathrm{CH}_{3} \mathrm{COOH}$ concentration just by coincidence starts to increase along with large N-bearing species. Given the criteria set above, it is intriguing that $\mathrm{CH}_{3} \mathrm{COOH}$ is detected toward I16293A given there was no clear evidence of any emission from $\mathrm{CH}_{3} \mathrm{CH}_{2} \mathrm{CN}$. Further higher sensitivity observations are once again necessary to confirm this detection and determine the evolutionary stage of the I16293A and B cores.

\section{4. $\mathrm{CH}_{3} \mathrm{COOH}$ Formation}

Both gas phase and grain surface chemistry undoubtedly play important roles in hot cores (e.g. Hasegawa et al. 1992; Garrod \& Herbst 2006; Garrod et al. 2008). Large molecule abundances can be enhanced enormously by grain surface chemistry while current models of gas phase chemistry alone are not sufficient to match observations. Garrod \& Herbst (2006) have demonstrated that radicals in the icy mantles on grain surfaces can move around as the temperature gradually increases from 10 to $200 \mathrm{~K}$ in star forming regions. Grain surfaces become efficient environments for large molecules to form. However, the details of $\mathrm{CH}_{3} \mathrm{COOH}$ formation in hot cores still remain unclear. The correlation between $\mathrm{CH}_{3} \mathrm{COOH}$ and large N-bearing molecules may suggest that some N-bearing molecules act as catalysts to the $\mathrm{CH}_{3} \mathrm{COOH}$ formation. The chemical and physical evolution timescales of star formation lacks direct observational evidence to confirm chemical models. Without the support of observation, it is difficult to establish solid chemical models. Garrod et al. (2008) have reported their new chemical model for $\mathrm{CH}_{3} \mathrm{COOH}$ in hot cores. Grain surface reactions have been enhanced to match observations. They show the time scales of hot cores warming up affect the abundances of the secondary radical $\mathrm{CH}_{3} \mathrm{CO}$, which can react with $\mathrm{OH}$ to form $\mathrm{CH}_{3} \mathrm{COOH}$. This effect can also cause the dissimilar abundances of structural isomers, which is consistent with our survey.

\subsection{Identifying Weak Spectral Features Using Model Spectra.}

When $\mathrm{CH}_{3} \mathrm{COOH}$ was first detected (Mehringer et al. 1997), it emphasized the importance of interferometry for the study of astrochemistry in hot cores. Star forming regions generally contain many regions of compact molecular emission. Although G19 has a complex structure, including extended and compact components (see Furuya et al. 2005), the $\mathrm{CH}_{3} \mathrm{COOH}$ detection 
toward G19 was conducted with a high resolution beam, $1^{\prime \prime} 9 \times 1$ 1.' 6 , which is the smallest used in a $\mathrm{CH}_{3} \mathrm{COOH}$ survey to date. The three observed molecules in $\mathrm{G} 19, \mathrm{CH}_{3} \mathrm{COOH}, \mathrm{HCOOCH}_{3}$ and $\mathrm{CH}_{3} \mathrm{CH}_{2} \mathrm{CN}$, are mainly located at the same component, which agrees with the $\mathrm{CH}_{3} \mathrm{CH}_{2} \mathrm{CN}$ observation by Furuya et al. (2005). As we continue extending $\mathrm{CH}_{3} \mathrm{COOH}$ surveys to smaller hot cores, the required resolution of observations will be higher.

Higher resolution observations also have the advantage of reducing the effect of line confusion present in a spectrum. Also, if the source size of the emitting region is well coupled to the synthesized beam of the array, weak spectral features that are lost due to beam dilution effects from single dish observations can be significantly resolved out of the noise. Thus, many new molecule detections over the last several years have used both a combination of single dish and array observations to confirm the detection (e.g., Belloche et al. 2008, 2009). In addition, these detections routinely use model spectra based on the laboratory and calculated rotational spectroscopy of energy levels and line strengths for molecular transitions, the physical conditions of the emitting regions including temperature and density and finally, the characteristics of the telescope including forward beam efficiency and field of view (i.e. beam size). Given the model spectra, the weak transitions of molecular species appear statistically significant in the observed spectrum. However, the model data have near infinite spectral resolution compared to the observed data and do not include an estimate of the noise level in the passband. These types of models are shown in the spectral line data presented in this work (red Gaussian traces in the first row of spectra in Figures 2, 4, 6 and 7).

In order to correctly ascertain whether a weak feature in the observed spectrum is due to a calculated line of a molecular species, we advocate taking the model data and convolving it with the spectral resolution of the instrument and adding the appropriate amount of random noise to the resultant spectrum to give a more accurate representation of the data collected by the telescope. The noise level is based on the measured noise of line free channels in the observed data. These modeled data are then subtracted from the observed data to form a residual spectrum. From these residuals, with the caveat of an accurate model of the physical conditions of the source, one can identify the remaining weak spectral features and determine if features close to the $1-2 \sigma$ level are in fact statistically significant spectral line features.

Based on the residual passbands shown in the last row of Figures 2, 4, 6 and 7, we include in Tables 3, 4 and 5 the possibility of unidentified lines (i.e. U-lines) in the data toward G19, G29 and IRAS 16293-2422. In addition, given the spectral line fit (red Gaussian traces) toward IRAS 16293-2422, there is a suggestion that the weak emission features shown toward I16293B may in fact be due to $\mathrm{CH}_{3} \mathrm{COOH}$. However, when this model spectrum is convolved with the noise level in the passband, these weak features completely disappear and there are definitely no statistically significant feature seen in the residual passbands. As a result, while we claim that the emission features that are coincident with the $\mathrm{CH}_{3} \mathrm{COOH}$ transitions toward I16293A are in fact from $\mathrm{CH}_{3} \mathrm{COOH}$, these features are only barely above the noise level so further high sensitivity observations are needed for a definitive detection. 
It is our recommendation that if model spectra are going to be the new proxy for identifying weak spectral features in observational data, the model data should be first convolved to the spectral resolution of the telescope and the appropriate amount of noise be added to the model in order to make an accurate representation as possible to the observed data. Only then can the 1-2 $\sigma$ spectral features be corrected assessed as statistically significant features in the observed data and not an artifact of the noise and spectral resolution.

\section{SUMMARY}

We have conducted a high resolution acetic acid $\left(\mathrm{CH}_{3} \mathrm{COOH}\right)$ survey at $3 \mathrm{~mm}$ wavelengths for the first time with CARMA towards two high-mass hot cores, G19.61-0.23 and G29.96-0.02, and a low-mass embedded protostar system, IRAS 16293-2422. We have detected $\mathrm{CH}_{3} \mathrm{COOH}$ emission in G19.61-0.23, via the two $\mathrm{CH}_{3} \mathrm{COOH}$ rotational transitions, $10_{*, 10}-9_{*, 9} E$ and $A$. While G19.610.23 consists of several clumps, high resolution mapping reveals that the $\mathrm{CH}_{3} \mathrm{COOH}$ emission is extremely compact $\left(<2^{\prime \prime}\right)$ toward component $\mathrm{C}$. Methyl formate $\left(\mathrm{HCOOCH}_{3}\right)$ and ethyl cyanide $\left(\mathrm{CH}_{3} \mathrm{CH}_{2} \mathrm{CN}\right)$ were also detected. The emission from these two molecules is more extended than the $\mathrm{CH}_{3} \mathrm{COOH}$ emission but the peaks of all three emission spectra are toward component $\mathrm{C}$. The $\mathrm{CH}_{3} \mathrm{COOH}$ column density and abundance ratio with respect to methyl formate $\left(\mathrm{HCOOCH}_{3}\right)$, are $2.0(1.0) \times 10^{16} \mathrm{~cm}^{-2}$ and $2.2(0.1) \times 10^{-1}$ respectively, which is higher but comparable to the other high-mass $\mathrm{CH}_{3} \mathrm{COOH}$ sources given the current level of detection. Although acetic acid is not detected toward G29.96-0.02, we have detected $\mathrm{HCOOCH}_{3}$ and $\mathrm{CH}_{3} \mathrm{CH}_{2} \mathrm{CN}$. The $\mathrm{HCOOCH}_{3}$ and $\mathrm{CH}_{3} \mathrm{CH}_{2} \mathrm{CN}$ emission features of G29.96-0.02 are weaker than G19.61-0.23 by a factor of 3. While we detect $\mathrm{CH}_{3} \mathrm{COOH}$ in G19.61-0.23, the non-detection of $\mathrm{CH}_{3} \mathrm{COOH}$ in G29.96-0.02 may be due to the limited sensitivity. Finally we have detected two new transitions of $\mathrm{HCOOCH}_{3}, 9_{2,8}-8_{2,7} E$ and $A$ toward IRAS 16293-2422 and weak emission features at the $2 \sigma$ level of $\mathrm{CH}_{3} \mathrm{COOH}$ toward I16293A. This is the first observation of new spectral features of $\mathrm{CH}_{3} \mathrm{COOH}$ since the reported detection by Cazaux et al. (2003). The $\mathrm{HCOOCH}_{3}$ spectrum toward I16293B shows narrower and more distinguishable line profiles than the spectrum toward I16293A, which may be suffering from self-absorption. However, we did not detect $\mathrm{CH}_{3} \mathrm{CH}_{2} \mathrm{CN}$ in IRAS 16293-2422 at the the $1 \sigma$ detection limit.

The new $\mathrm{CH}_{3} \mathrm{COOH}$ detection demonstrates the strong correlation between large $\mathrm{O}$ and $\mathrm{N}$ bearing species and $\mathrm{CH}_{3} \mathrm{COOH}$ in hot cores. The timescale of hot cores may also play a role in $\mathrm{O} / \mathrm{N}$ chemical differentiation and hence $\mathrm{CH}_{3} \mathrm{COOH}$ formation. However, we need more $\mathrm{CH}_{3} \mathrm{COOH}$ detections to confirm these suggestions. The compactness of the emission from large molecules stresses the need of interferometers for hot core chemistry observations. Our high resolution survey has provided critical information about the chemical and physical properties of hot cores, which can be used to constrain the hot core chemical models. Higher resolution observations are still required to precisely determine temperature and the density distribution of large $\mathrm{O}$ and $\mathrm{N}$-bearing species for physical-parameter sensitive chemical models. Through well-improved chemical models, 
we can better understand the distinctive hot core chemistry.

We especially would like to thank Francesco Fontani, Paolo Caselli and Friedrich Wyrowski for providing the IRAM 30-m data presented in Figure 8. It is with this type of collaboration that we can truly make advances in understanding the formation of complex molecules in astronomical environments. We also thank an anonymous referee for some very useful comments that undoubtedly strengthen the manuscript. We acknowledge support from the Laboratory for Astronomical Imaging at the University of Illinois and NSF grant AST-0540459. Support for CARMA construction was derived from the Gordon and Betty Moore Foundation, the Kenneth T. and Eileen L. Norris Foundation, the Associates of the California Institute of Technology, the states of California, Illinois, and Maryland, and the National Science Foundation. Ongoing CARMA development and operations are supported by the National Science Foundation under a cooperative agreement, and by the CARMA partner universities. This work was also supported in part by the NSF Centers for Chemical Innovation through award CHE-0847919. 


\section{REFERENCES}

Belloche, A., Garrod, R. T., Müller, H. S. P., Menten, K. M., Comito, C., \& Schilke, P. 2009, A\&A, 499,215

Belloche, A., Menten, K. M., Comito, C., Müller, H. S. P., Schilke, P., Ott, J., Thorwirth, S., \& Hieret, C. 2008, A\&A, 492, 769

Beuther, H., Zhang, Q., Bergin, E. A., Sridharan, T. K., Hunter, T. R., \& Leurini, S. 2007, A\&A, 468, 1045

Blake, G. A., Sutton, E. C., Masson, C. R., \& Phillips, T. G. 1987, ApJ, 315, 621

Bottinelli, S., et al. 2004, ApJ, 617, L69

Cazaux, S., Tielens, A. G. G. M., Ceccarelli, C., Castets, A., Wakelam, V., Caux, E., Parise, B., \& Teyssier, D. 2003, ApJ, 593, L51

Cesaroni, R., Hofner, P., Walmsley, C. M., \& Churchwell, E. 1998, A\&A, 331, 709

Chandler, C. J., Brogan, C. L., Shirley, Y. L., \& Loinard, L. 2005, ApJ, 632, 371

Di Francesco, J., Myers, P. C., Wilner, D. J., Ohashi, N., \& Mardones, D. 2001, ApJ, 562, 770

Fontani, F., Pascucci, I., Caselli, P., Wyrowski, F., Cesaroni, R., \& Walmsley, C. M. 2007, A\&A, 470,639

Friedel, D. N., Snyder, L. E., Turner, B. E., \& Remijan, A. 2004, ApJ, 600, 234

Fukuyama, Y., Odashima, H., Takagi, K., \& Tsunekawa, S. 1996, ApJS, 104, 329

Furuya, R. S., Cesaroni, R., Takahashi, S., Momose, M., Testi, L., Shinnaga, H., \& Codella, C. 2005, ApJ, 624, 827

Garay, G., Moran, J. M., Rodriguez, L. F., \& Reid, M. J. 1998, ApJ, 492, 635

Garay, G., Reid, M. J., \& Moran, J. M. 1985, ApJ, 289, 681

Garrod, R. T., \& Herbst, E. 2006, A\&A, 457, 927

Garrod, R. T., Weaver, S. L. W., \& Herbst, E. 2008, ApJ, 682, 283

Gaume, R. A., Claussen, M. J., de Pree, C. G., Goss, W. M., \& Mehringer, D. M. 1995, ApJ, 449, 663

Genzel, R., \& Downes, D. 1977, A\&AS, 30, 145

Goldsmith, P. F., \& Langer, W. D. 1999, ApJ, 517, 209 
Guélin, M., Brouillet, N., Cernicharo, J., Combes, F., \& Wooten, A. 2008, Ap\&SS, 313, 45

Hasegawa, T. I., Herbst, E., \& Leung, C. M. 1992, ApJS, 82, 167

Hofner, P., Wyrowski, F., Walmsley, C. M., \& Churchwell, E. 2000, ApJ, 536, 393

Ilyushin, V., Kleiner, I., \& Lovas, F. J. 2008, Phys. Chem. Ref. Data, 37, 97

Kalenskii, S. V., Berulis, I. I., Val'tts, I. E., Dzura, A. M., Slysh, V. I., \& Vasil'kov, V. I. 1994, AZh, 71, 51

Kuan, Y.-J., et al. 2004, ApJ, 616, L27

Larionov, G. M., Val'tts, I. E., Winnberg, A., Johansson, L. E. B., Booth, R. S., \& Golubev, V. V. 1999, A\&AS, 139, 257

Loinard, L., Chandler, C. J., Rodríguez, L. F., D’Alessio, P., Brogan, C. L., Wilner, D. J., \& Ho, P. T. P. 2007, ApJ, 670, 1353

Looney, L. W., Mundy, L. G., \& Welch, W. J. 2000, ApJ, 529, 477

Matthews, H. E., Goss, W. M., Winnberg, A., \& Habing, H. J. 1977, A\&A, 61, 261

Mehringer, D. M., Snyder, L. E., Miao, Y., \& Lovas, F. J. 1997, ApJ, 480, L71

Miao, Y., Mehringer, D. M., Kuan, Y.-J., \& Snyder, L. E. 1995, ApJ, 445, L59

Müller, H. S. P., Schlöder, F., Stutzki, J., \& Winnewisser, G. 2005, Journal of Molecular Structure, 742,215

Oesterling, L. C., Albert, S., De Lucia, F. C., Sastry, K. V. L. N., \& Herbst, E. 1999, ApJ, 521, 255

Olmi, L., Cesaroni, R., Hofner, P., Kurtz, S., Churchwell, E., \& Walmsley, C. M. 2003, A\&A, 407, 225

Remijan, A., Shiao, Y.-S., Friedel, D. N., Meier, D. S., \& Snyder, L. E. 2004, ApJ, 617, 384

Remijan, A., Snyder, L. E., Friedel, D. N., Liu, S.-Y., \& Shah, R. Y. 2003, ApJ, 590, 314

Remijan, A., Snyder, L. E., Liu, S.-Y., Mehringer, D., \& Kuan, Y.-J. 2002, ApJ, 576, 264

Remijan, A. J., \& Hollis, J. M. 2006, ApJ, 640, 842

Remijan, A. J., Markwick-Kemper, A., \& ALMA Working Group on Spectral Line Frequencies. 2007, in Bulletin of the American Astronomical Society, Vol. 38, Bulletin of the American Astronomical Society, 963

Rodgers, S. D., \& Charnley, S. B. 2001, ApJ, 546, 324 
Sault, R. J., Teuben, P. J., \& Wright, M. C. H. 1995, in Astronomical Society of the Pacific Conference Series, Vol. 77, Astronomical Data Analysis Software and Systems IV, ed. R. A. Shaw, H. E. Payne, \& J. J. E. Hayes, 433

Schöier, F. L., Jørgensen, J. K., van Dishoeck, E. F., \& Blake, G. A. 2002, A\&A, 390, 1001

Shirley, Y. L., Evans, II, N. J., Young, K. E., Knez, C., \& Jaffe, D. T. 2003, ApJS, 149, 375

Snyder, L. E. 2006, Proceedings of the National Academy of Science, 103, 12243

Snyder, L. E., Kuan, Y., \& Miao, Y. 1994, in Lecture Notes in Physics, Berlin Springer Verlag, Vol. 439, The Structure and Content of Molecular Clouds, ed. T. L. Wilson \& K. J. Johnston, 187

Snyder, L. E., et al. 2005, ApJ, 619, 914

Sutton, E. C., Peng, R., Danchi, W. C., Jaminet, P. A., Sandell, G., \& Russell, A. P. G. 1995, ApJS, 97, 455

Wootten, A. 1989, ApJ, 337, 858

Wu, J., \& Evans, II, N. J. 2003, ApJ, 592, L79

Wu, Y., Qin, S., Guan, X., Xue, R., Ren, Z., Liu, T., Huang, M., \& Chen, S. 2009, ApJ, 697, L116

Wyrowski, F., Schilke, P., Walmsley, C. M., \& Menten, K. M. 1999, ApJ, 514, L43 
Table 1. Observed Sources

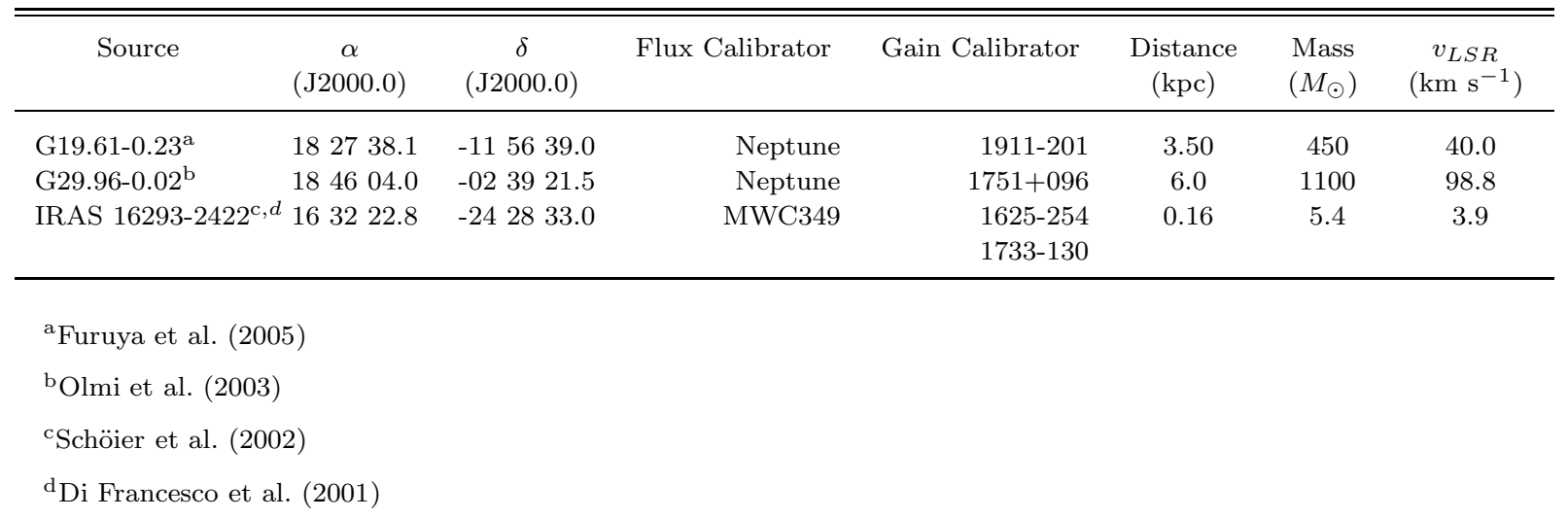

Table 2. OBSERVED MOLECULES AND TRANSITIONS

\begin{tabular}{cccccc}
\hline \hline \multirow{2}{*}{ Species } & Rotational Partition Function & Transition & Frequency $(\mathrm{MHz})$ & $E_{u}(\mathrm{~K})$ & $<S_{j, j} \mu^{2}>\left(\mathrm{debye}^{2}\right)$ \\
\hline \multirow{2}{*}{$\mathrm{CH}_{3} \mathrm{COOH}^{\mathrm{a}}$} & \multirow{2}{*}{$Q_{r}=14.1 T_{r}^{3 / 2}$} & $10_{*, 10}-9_{*, 9} E$ & $111,507.270(20)$ & 30.5 & 54.8 \\
& & $10_{*, 10}-9_{*, 9} A$ & $111,548.533(20)$ & 30.5 & 54.8 \\
$\mathrm{HCOOCH}_{3}{ }^{\mathrm{b}}$ & $Q_{r}=12.45 T_{r}^{3 / 2}$ & $9_{2,8}-8_{2,7} E$ & $107,537.189(25)$ & 28.8 & 22.8 \\
& & $9_{2,8}-8_{2,7} A$ & $107,543.746(25)$ & 28.8 & 22.8 \\
$\mathrm{CH}_{3} \mathrm{CH}_{2} \mathrm{CN}^{\mathrm{c}}$ & $Q_{r}=7.17 T_{r}^{3 / 2}$ & $12_{11,2}-11_{11,1}$ & $107,539.854(3)$ & 167.8 & 28.4 \\
& & $12_{11,1}-11_{11,0}$ & $107,539.854(3)$ & 167.8 & 28.4 \\
& & $124,9-11_{4,8}$ & $107,543.918(3)$ & 51.4 & 158.1 \\
& & $12_{4,8}-11_{4,7}$ & $107,547.593(3)$ & 51.4 & 158.1 \\
& $12_{3,10}-11_{3,9}$ & $107,594.040(3)$ & 43.6 & 166.8 \\
\hline
\end{tabular}

Note. - The $1 \sigma$ uncertainty of the frequencies is in units of $\mathrm{kHz}$. Each of the $\mathrm{CH}_{3} \mathrm{COOH}$ lines consists of two a-type and two b-type transitions. This is represented by an asterisk substituted for the $\mathrm{K}_{-}$quantum numbers.

Ilyushin et al. (2008)

Oesterling et al. (1999)

[Fukuvama et al. (1996); Remiian et al. (2007) 
Table 3. DETECTED MOLECULES AND TRANSITIONS TOWARD G19.61-0.23

\begin{tabular}{cccccc}
\hline \hline Species & Transition & $\Delta \mathrm{I}\left(\mathrm{Jy} \mathrm{beam}{ }^{-1}\right)$ & $\Delta v\left(\mathrm{~km} \mathrm{~s}^{-1}\right)$ & $\Delta \mathrm{I}\left(\mathrm{Jy} \mathrm{beam}^{-1}\right)$ & $\Delta v\left(\mathrm{~km} \mathrm{~s}^{-1}\right)$ \\
\hline \multicolumn{5}{c}{ Fitted Data } & Model Data \\
\hline $\mathrm{CH}_{3} \mathrm{COOH}$ & $10_{*, 10}-9_{*, 9} E$ & $0.07 \pm 0.01$ & $6.4 \pm 0.9$ & 0.10 & 6.4 \\
& $10_{*, 10}-9_{*, 9} A$ & $0.09 \pm 0.02$ & $6.4 \pm 0.9$ & 0.12 & 6.4 \\
$\mathrm{HCOOCH}_{3}$ & $9_{2,8}-8_{2,7} E$ & $0.20 \pm 0.02$ & $6.4 \pm 0.9$ & 0.18 & 6.4 \\
& $9_{2,8}-82,7$ & $0.20 \pm 0.02$ & $6.4 \pm 0.9$ & 0.18 & 6.4 \\
$\mathrm{CH}_{3} \mathrm{CH}_{2} \mathrm{CN}$ & $12_{11, *}-11_{11, *}$ & $0.18 \pm 0.02$ & $12.9 \pm 0.4$ & 0.13 & 12.9 \\
& $124,9-11_{4,8}$ & $0.59 \pm 0.02$ & $12.9 \pm 0.4$ & 0.66 & 12.9 \\
& $124,8-11_{4,7}$ & $0.59 \pm 0.02$ & $12.9 \pm 0.4$ & 0.66 & 12.9 \\
$\mathrm{U} 107.588$ & $12,10-11_{3,9}$ & $0.71 \pm 0.01$ & $12.9 \pm 0.4$ & 0.72 & \\
$\mathrm{U} 107.591$ & $107.5880 \mathrm{GHz}$ & $0.16 \pm 0.04$ & $5.6 \pm 1.6$ & & \\
$\mathrm{U} 107.597$ & $107.5910 \mathrm{GHz}$ & $0.27 \pm 0.03$ & $7.8 \pm 1.2$ & & \\
$\mathrm{U} 107.604$ & $107.5970 \mathrm{GHz}$ & $0.31 \pm 0.03$ & $12.5 \pm 1.2$ & & \\
$\mathrm{U} 111.506$ & $107.6040 \mathrm{GHz}$ & $0.23 \pm 0.03$ & $11.1 \pm 1.5$ & & \\
$\mathrm{U} 111.510$ & $111.5055 \mathrm{GHz}$ & $0.06 \pm 0.02$ & $5.2 \pm 1.7$ & & \\
$\mathrm{U} 111.546$ & $111.5095 \mathrm{GHz}$ & $0.07 \pm 0.01$ & $17.2 \pm 4.5$ & & \\
\hline
\end{tabular}

Note. - The $\mathrm{HCOOCH}_{3}$ lines were fit assuming the same linewidth. The same line width fitting criteria applies for the $\mathrm{CH}_{3} \mathrm{CH}_{2} \mathrm{CN}$ and $\mathrm{CH}_{3} \mathrm{COOH}$ lines. For simplicity, the two $\mathrm{CH}_{3} \mathrm{CH}_{2} \mathrm{CN}$ lines listed in Table 2 are now represented as one by an asterisk substituted for the $\mathrm{K}_{-}$quantum numbers. The other three $\mathrm{CH}_{3} \mathrm{CH}_{2} \mathrm{CN}$ lines remain unchanged.

Table 4. DETECTED MOLECULES AND TRANSITIONS TOWARD G29.96-0.02

\begin{tabular}{cccccc}
\hline \hline Species & Transition & $\Delta \mathrm{I}\left(\mathrm{Jy} \mathrm{beam}^{-1}\right)$ & $\Delta v\left(\mathrm{~km} \mathrm{~s}^{-1}\right)$ & $\Delta \mathrm{I}\left(\mathrm{Jy} \mathrm{beam}^{-1}\right)$ & $\Delta v\left(\mathrm{~km} \mathrm{~s}^{-1}\right)$ \\
\hline \multicolumn{5}{c}{ Fitted Data } & Model Data \\
\hline $\mathrm{CH}_{3} \mathrm{COOH}$ & $10_{*, 10}-9_{*, 9} E$ & $\sim 0.04$ & & \\
& $10_{*, 10}-9_{*, 9} A$ & $<0.03$ & & 0.09 & 7.8 \\
$\mathrm{HCOOCH}_{3}$ & $9_{2,8}-8_{2,7} E$ & $0.08 \pm 0.01$ & $7.8 \pm 1.1$ & 0.09 & 10.8 \\
& $9_{2,8}-8_{2,7} A$ & $0.08 \pm 0.01$ & $7.8 \pm 1.1$ & 0.03 & 10.8 \\
$\mathrm{CH}_{3} \mathrm{CH}_{2} \mathrm{CN}$ & $12_{11, *}-11_{11, *}$ & $0.07 \pm 0.01$ & $10.8 \pm 0.4$ & 0.23 & 10.8 \\
& $12_{4,9}-11_{4,8}$ & $0.21 \pm 0.01$ & $10.8 \pm 0.4$ & 0.26 & \\
$\mathrm{U} 107.591$ & $12_{4,8}-11_{4,7}$ & $0.21 \pm 0.01$ & $10.8 \pm 0.4$ & & \\
$\mathrm{U} 107.597$ & $12{ }_{3,10}-11_{3,9}$ & $0.27 \pm 0.01$ & $10.8 \pm 0.4$ & & \\
$\mathrm{U} 107.604$ & $107.5910 \mathrm{GHz}$ & $0.11 \pm 0.01$ & $8.5 \pm 0.9$ & & \\
$\mathrm{U} 111.541$ & $107.5975 \mathrm{GHz}$ & $0.08 \pm 0.01$ & $10.8 \pm 1.2$ & & \\
\hline
\end{tabular}

Note. - Table comments are the same as in Table 3. 
Table 5. DETECTED MOLECULES AND TRANSITIONS TOWARD IRAS 16293-2422

\begin{tabular}{|c|c|c|c|c|c|}
\hline Species & Transition & $\Delta \mathrm{I}\left(\mathrm{Jy} \mathrm{beam}^{-1}\right)$ & $\Delta v\left(\mathrm{~km} \mathrm{~s}^{-1}\right)$ & $\Delta \mathrm{I}\left(\mathrm{Jy} \mathrm{beam}^{-1}\right)$ & $\Delta v\left(\mathrm{~km} \mathrm{~s}^{-1}\right)$ \\
\hline & & \multicolumn{2}{|c|}{ Fitted Data } & \multicolumn{2}{|c|}{ Model Data } \\
\hline \multicolumn{6}{|c|}{ IRAS 16293-2422 A } \\
\hline \multirow[t]{2}{*}{$\mathrm{CH}_{3} \mathrm{COOH}$} & $10_{*, 10}-9_{*, 9} E$ & $0.05 \pm 0.01$ & $6.0 \pm 2.0$ & 0.03 & 6 \\
\hline & $10_{*, 10}-9_{*, 9} A$ & $0.05 \pm 0.01$ & $6.0 \pm 2.0$ & 0.05 & 6 \\
\hline \multirow[t]{2}{*}{$\mathrm{HCOOCH}_{3}$} & $9_{2,8}-8_{2,7} E$ & $0.050 \pm 0.009$ & $14.6 \pm 1.5$ & 0.049 & 14.6 \\
\hline & $9_{2,8}-8_{2,7} A$ & $0.050 \pm 0.008$ & $14.6 \pm 1.5$ & 0.049 & 14.6 \\
\hline U111.508 & $111.5080 \mathrm{GHz}$ & $0.05 \pm 0.01$ & $5.0 \pm 2.0$ & & \\
\hline \multicolumn{6}{|c|}{ IRAS $16293-2422 \mathrm{~B}$} \\
\hline \multirow[t]{2}{*}{$\mathrm{CH}_{3} \mathrm{COOH}$} & $10_{*, 10}-9_{*, 9} E$ & $<0.01$ & & & \\
\hline & $10_{*, 10}-9_{*, 9} A$ & $<0.01$ & & & \\
\hline \multirow[t]{2}{*}{$\mathrm{HCOOCH}_{3}$} & $9_{2,8}-8_{2,7} E$ & $0.063 \pm 0.005$ & $5.5 \pm 0.5$ & 0.059 & 5.5 \\
\hline & $9_{2,8}-8_{2,7} A$ & $0.063 \pm 0.005$ & $5.5 \pm 0.5$ & 0.059 & 5.5 \\
\hline $\mathrm{U} 111.510$ & $111.5095 \mathrm{GHz}$ & $0.06 \pm 0.01$ & $8.0 \pm 2.0$ & & \\
\hline U111.541 & $111.5410 \mathrm{GHz}$ & $0.06 \pm 0.01$ & $5.0 \pm 1.0$ & & \\
\hline
\end{tabular}

Table 6. COLUMN DENSITIES OF THE OBSERVED MOLECULES

\begin{tabular}{lrrrr}
\hline \hline Species $\left(\mathrm{cm}^{-2}\right)$ & G19.61-0.23 & G29.96-0.02 & \multicolumn{1}{c}{ I16293A } & \multicolumn{1}{c}{ I16293B } \\
\hline $\mathrm{CH}_{3} \mathrm{COOH}$ & $(2.0 \pm 1.0) \times 10^{16}$ & $<9 \times 10^{14}$ & $\sim 1.6 \times 10^{15}$ & $<6 \times 10^{14}$ \\
$\mathrm{HCOOCH}_{3}$ & $(9.0 \pm 2.0) \times 10^{16}$ & $(4.0 \pm 1.0) \times 10^{16}$ & $(1.5 \pm 0.3) \times 10^{16}$ & $(6.0 \pm 1.0) \times 10^{15}$ \\
$\mathrm{CH}_{3} \mathrm{CH}_{2} \mathrm{CN}$ & $(6.0 \pm 3.0) \times 10^{16}$ & $(1.1 \pm 0.7) \times 10^{16}$ & $<6 \times 10^{13}$ & $<3 \times 10^{14}$ \\
& & & & \\
\hline
\end{tabular}

Table 7. COMPARISON OF $\mathrm{CH}_{3} \mathrm{COOH}$ SOURCES

\begin{tabular}{|c|c|c|c|c|c|}
\hline Species & Sgr B2(N-LMH $)^{\mathrm{a}}$ & $\mathrm{W} 51 \mathrm{e} 2^{\mathrm{b}}$ & $\mathrm{G} 34.3+0.15^{\mathrm{c}}$ & G19.61-0.23 ${ }^{\mathrm{d}}$ & IRAS $16293 \mathrm{~A}^{\mathrm{d}}$ \\
\hline $\mathrm{N}_{\mathrm{CH}_{3} \mathrm{COOH}}\left(\mathrm{cm}^{-2}\right)$ & $\sim 7.3 \times 10^{15}$ & $1.7 \pm 0.5 \times 10^{16}$ & $(0.77-1.64) \times 10^{15}$ & $2.0 \pm 1.0 \times 10^{16}$ & $\sim 1.6 \times 10^{15}$ \\
\hline $\mathrm{N}_{\mathrm{CH}_{3} \mathrm{COOH}} / \mathrm{N}_{\mathrm{HCOOCH}_{3}}$ & $(4-7) \times 10^{-2}$ & $(1-6) \times 10^{-2}$ & $\sim 3.3 \times 10^{-2}$ & $2.2 \pm 0.1 \times 10^{-1}$ & $1.0 \pm 0.1 \times 10^{-1}$ \\
\hline
\end{tabular}

Mehringer et al. (1997); $T_{r}=200 \mathrm{~K}$

Remijan et al. (2002); $T_{r}=201 \mathrm{~K}$

Remijan et al. (2003); $T_{r}=70-185 \mathrm{~K}$

$\mathrm{d}_{\text {this }}$ work; $T_{r}=161 \mathrm{~K}$ for G19 and $T_{r}=62 \mathrm{~K}$ for I16293A 

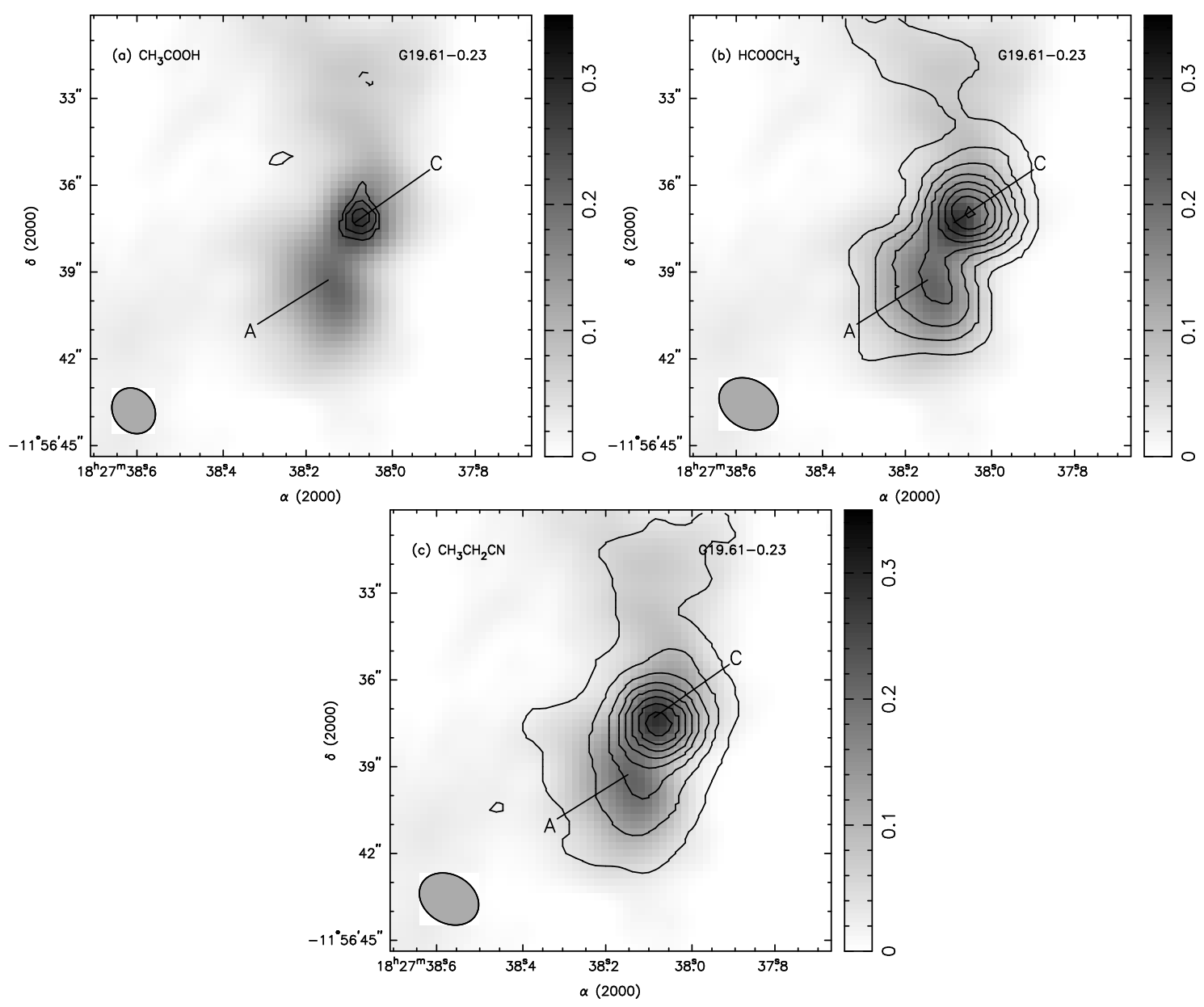

Fig. 1. - G19 maps. (a) $\mathrm{CH}_{3} \mathrm{COOH}$ contours overlaid on the gray continuum map. The continuum emission maps shown were made from channels contained in the wideband windows which were free

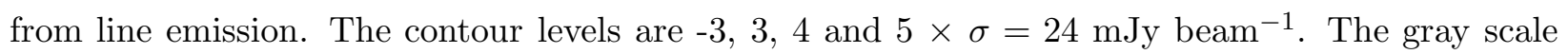
unit is Jy beam ${ }^{-1}$. The synthesized beam size is $1^{\prime \prime} 65 \times 1^{\prime \prime} .42$ (P.A. $=46.3^{\circ}$ ), shown in the bottom left corner. The continuum beam size is $1^{\prime \prime} .58 \times 1^{\prime \prime} .45\left(\right.$ P.A. $\left.=36.4^{\circ}\right)$. In the continuum map, there are two main components, $\mathrm{A}$ and $\mathrm{C}$. The $\mathrm{CH}_{3} \mathrm{COOH}$ emission is mainly overlaid on component $\mathrm{C}$. (b) $\mathrm{HCOOCH}_{3}$ contours overlaid on the gray continuum map. The contour levels averaged over 3 channels are $3,6,9,12,15,18,21,24,27$ and $30 \times \sigma=20$ mJy beam $^{-1}$. The synthesized beam size is $2^{\prime \prime} .16 \times 1^{\prime \prime} 67$ (P.A. $=59.6^{\circ}$ ). (c) $\mathrm{CH}_{3} \mathrm{CH}_{2} \mathrm{CN}$ contours overlaid on the gray continuum map. The contour levels averaged over 6 channels, are $3,9,15,21,27,33,39,45,51,57$ and $63 \times \sigma=18$ mJy beam ${ }^{-1}$. The synthesized beam size is $2^{\prime \prime} 16 \times 1^{\prime \prime} .67\left(\mathrm{P} . \mathrm{A} .=60.3^{\circ}\right)$. 

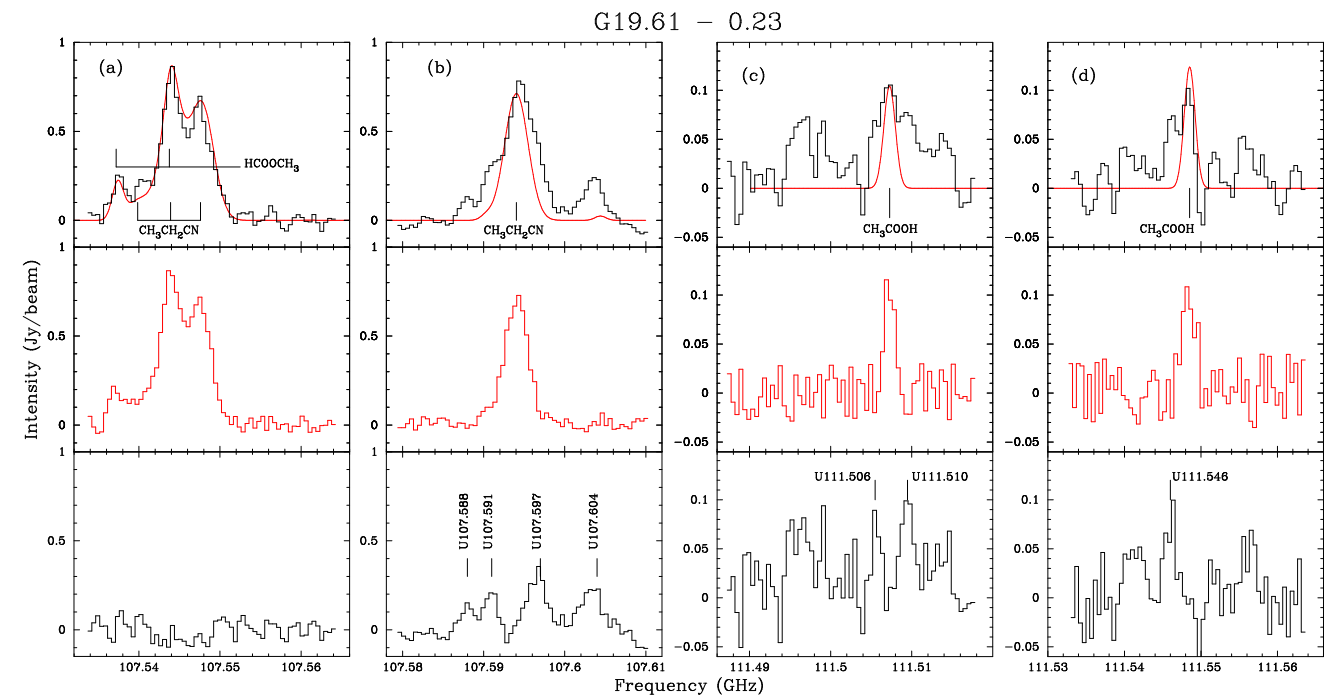

Fig. 2. - The observed spectra of G19.61-0.23. The first column (a) presents two $\mathrm{HCOOCH}_{3}$ lines, $9_{2,8}-8_{2,7} E$ and $9_{2,8}-8_{2,7} A$; and four $\mathrm{CH}_{3} \mathrm{CH}_{2} \mathrm{CN}$ lines, $12_{4,9}-11_{4,8}, 12_{4,8}-11_{4,7}, 12_{11,2}-11_{11,1}$ and $12_{11,1}-11_{11,0}$. The $12_{11,2}-11_{11,1}$ and $12_{11,1}-11_{11,0}$ lines are not spectrally resolved. The $\mathrm{v}_{L S R}$ range is -15 to $69.4 \mathrm{~km} \mathrm{~s}^{-1}$. The second column (b) contains only one known line, $\mathrm{CH}_{3} \mathrm{CH}_{2} \mathrm{CN}$ $12_{3,10}-11_{3,9}$. The $\mathrm{v}_{L S R}$ range is -3.4 to $81 \mathrm{~km} \mathrm{~s}^{-1}$. The third column (c) contains the $\mathrm{CH}_{3} \mathrm{COOH}$ $10_{*, 10}-9_{*, 9} E$ line and the fourth column (d) contains the $\mathrm{CH}_{3} \mathrm{COOH} 10_{*, 10}-9_{*, 9} A$ line. The rest frequencies of the windows are 107.54375, 107.59404, 111.50727 and $111.54853 \mathrm{GHz}$, respectively. The $\mathrm{v}_{L S R}$ ranges are 11.3 to 92.7 and -1.8 to $79.6 \mathrm{~km} \mathrm{~s}^{-1}$ for column (c) and (d), respectively. The first row presents the observed spectra (Hanning smoothed over 3 channels) overlaid on modeled spectral line data represented by Gaussians. The second row shows the modeled spectral line data from the first row convolved with the spectral resolution of the original observation and with random noise added to the spectrum. The value of the random noise is obtained with the line-free continuum spectrum in the windows. Finally, the third row shows the residuals from the modeled data in the second row subtracted from the observational data in the first row. 

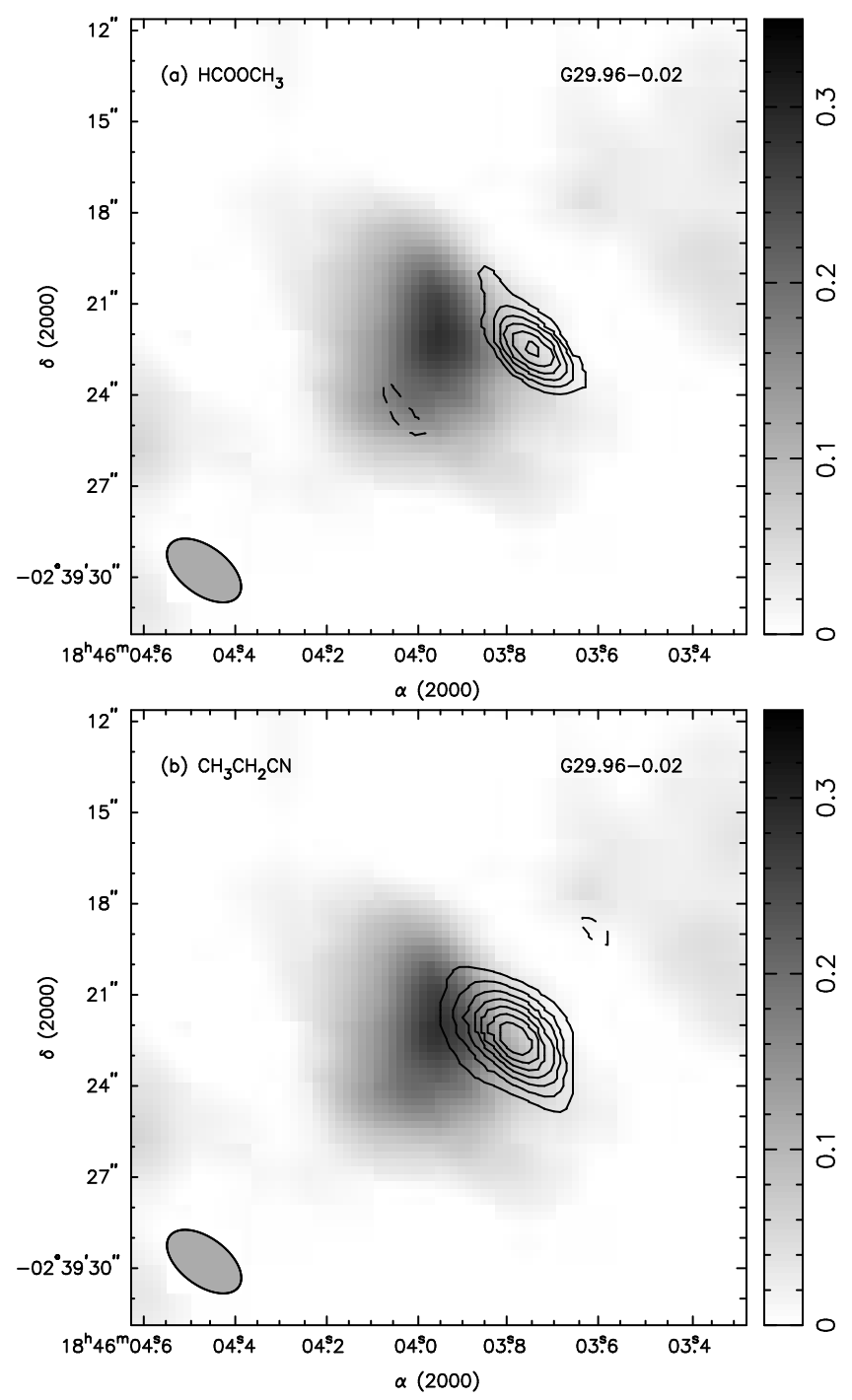

Fig. 3.- G29 maps. (a) $\mathrm{HCOOCH}_{3}$ contours overlaid on the gray continuum map. The contour levels averaged over 3 channels are $-3,3,4,5,6,7$ and $8 \times \sigma=21 \mathrm{mJy}^{-1}$ beam $^{-1}$. The synthesized beam size is $2^{\prime \prime} .81 \times 1^{\prime \prime} .56$ (P.A. $=53.2^{\circ}$ ), shown in the bottom left corner. (b) $\mathrm{CH}_{3} \mathrm{CH}_{2} \mathrm{CN}$ contours overlaid on the gray continuum map. The contour levels averaged over 6 channels, are $-3,3,5,7$, $9,11,13$ and $15 \times \sigma=16 \mathrm{mJy}^{\text {beam }}{ }^{-1}$. The synthesized beam size is $2^{\prime \prime} .82 \times 1^{\prime \prime} .56\left(\mathrm{P} . \mathrm{A} .=53.5^{\circ}\right)$. 


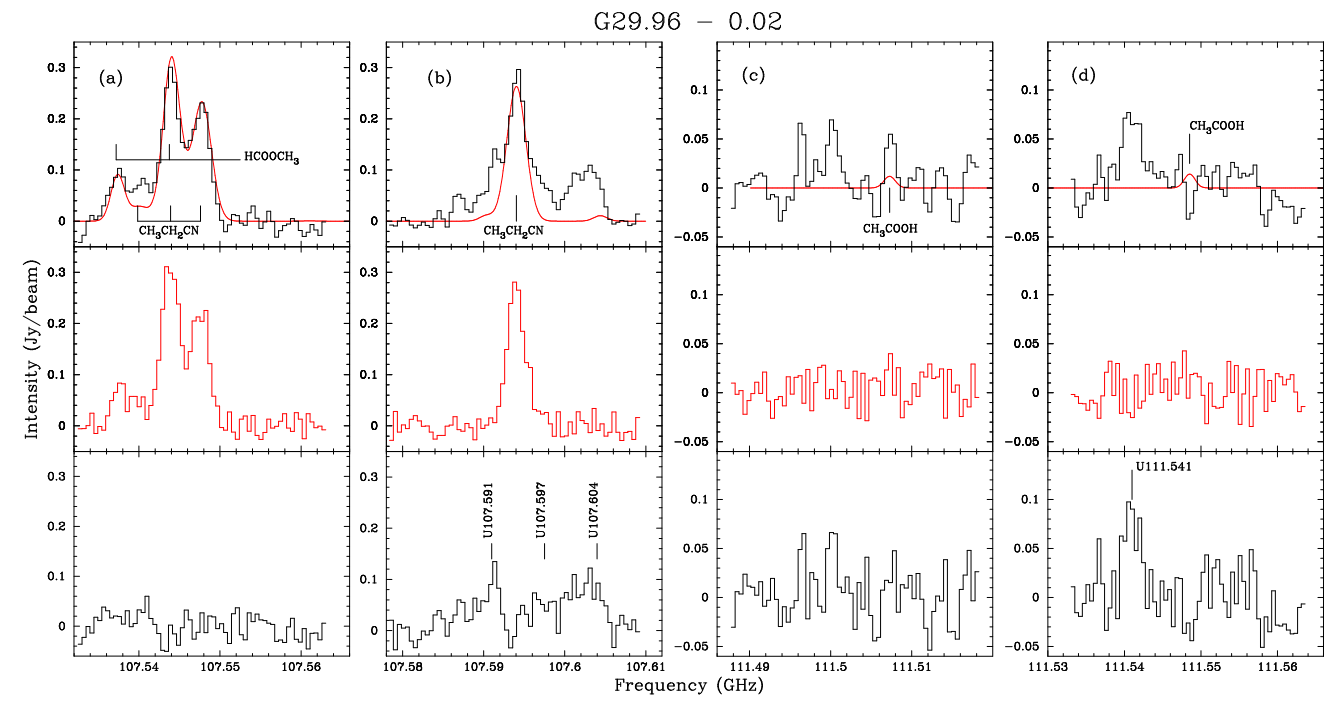

Fig. 4. - The observed spectra of G29.96-0.02. The arrangement of frequency and windows is the same as Figure 2. The $\mathrm{v}_{L S R}$ ranges are 44.6 to $129,56.2$ to $140.6,68.8$ to 150.2 and 55.7 to 137.1 $\mathrm{km} \mathrm{s}^{-1}$, respectively. 

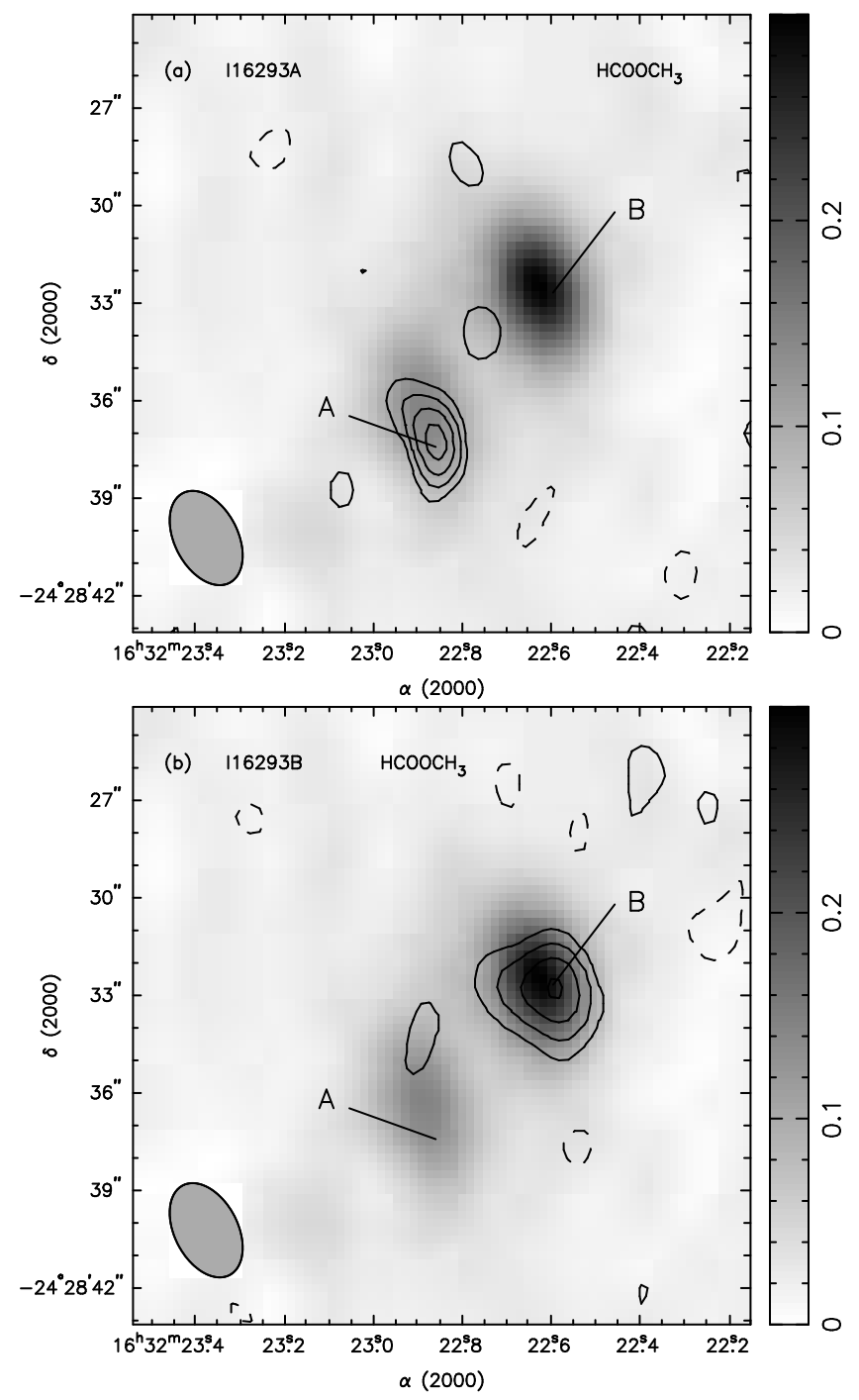

Fig. 5.- The $\mathrm{HCOOCH}_{3}$ contour maps are made individually for I16293A and B (denoted as A and $\mathrm{B}$ on the maps) due to the different linewidths. The contours are overlaid on the gray continuum map of IRAS 16293-2422. (a) The contours located at I16293A are made with the $9_{2,8}-8_{2,7} E$ line averaged over six channels. The contour levels are $-2,2,3,4$ and $5 \times \sigma=21$ mJy beam $^{-1}$. (b) The contours located at I16293B are made with one channel of the $9_{2,8}-8_{2,7} E$ line. The contour levels are $-2,2,3,4$ and $5 \times \sigma=25$ mJy beam $^{-1}$. 


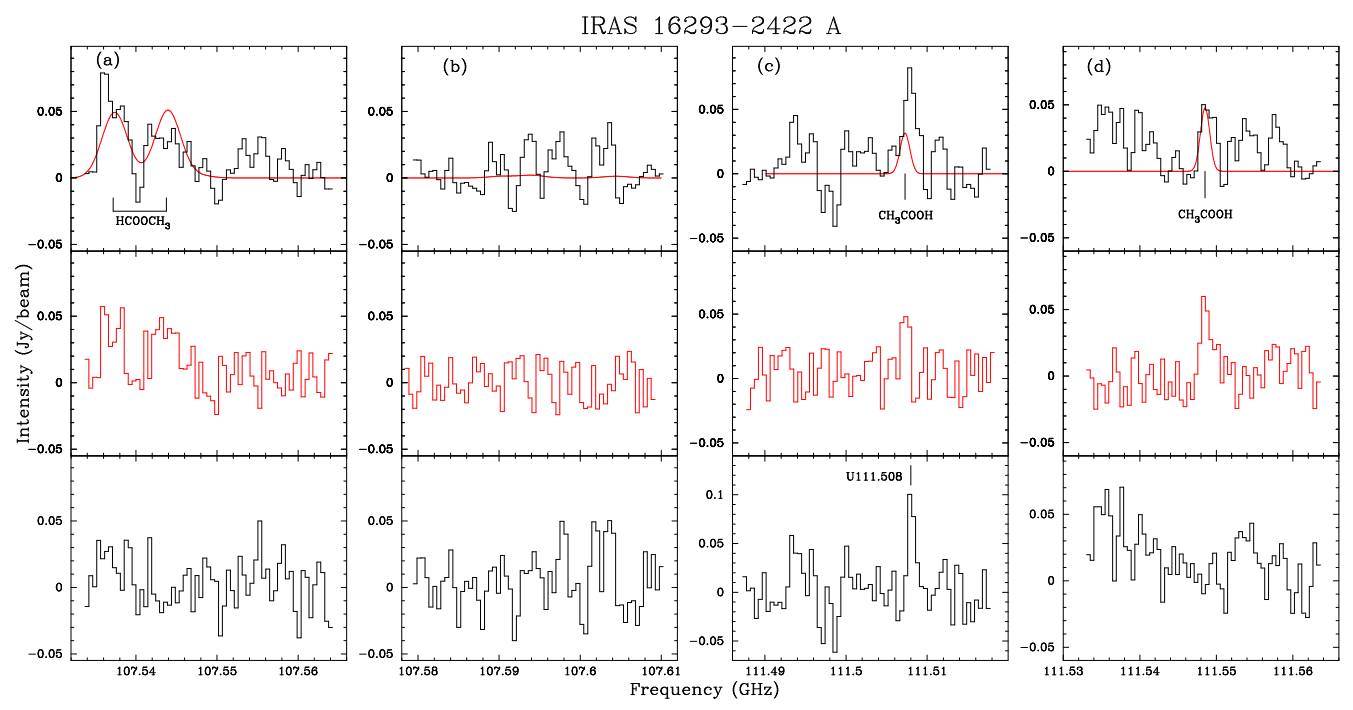

Fig. 6. - The observed spectra of I16293A. The arrangement of frequency and windows is the same as Figure 2. The column $\mathrm{v}_{L S R}$ ranges are 44.6 to $129,56.2$ to $140.6,68.8$ to 150.2 and 55.7 to 137.1 $\mathrm{km} \mathrm{s}^{-1}$, respectively.

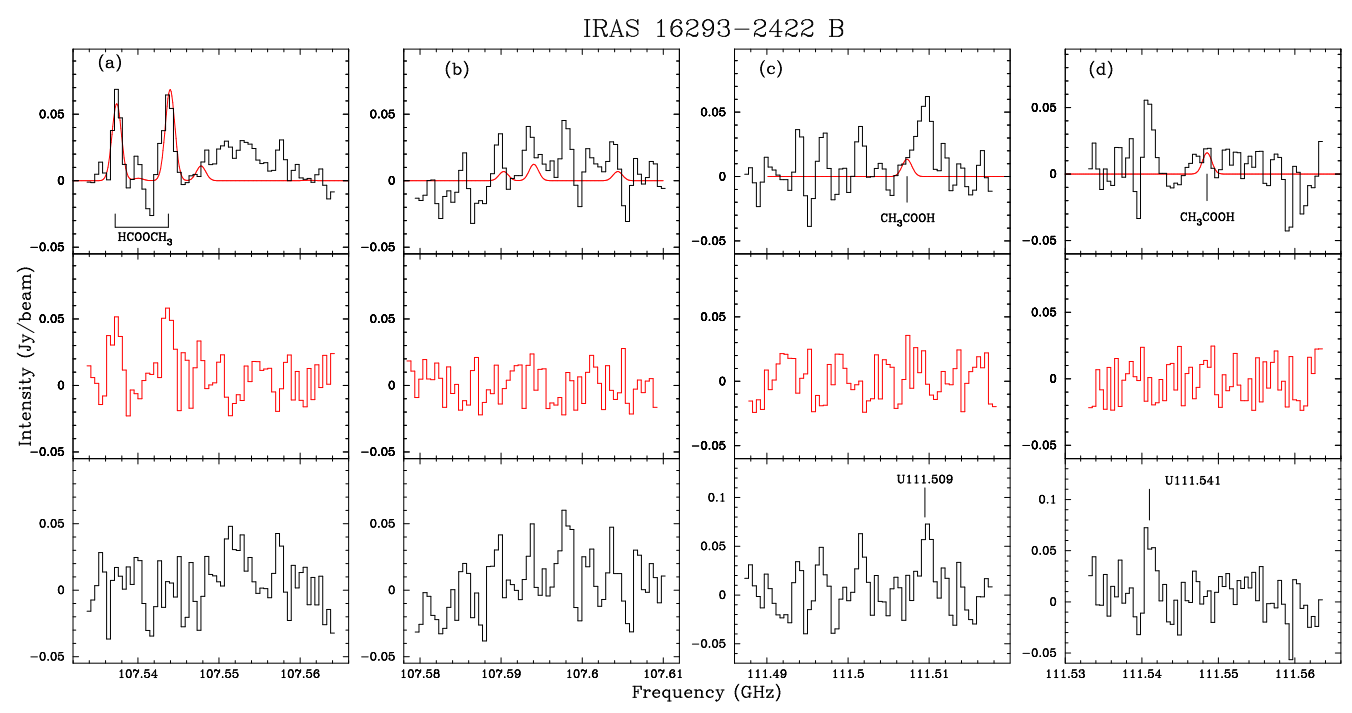

Fig. 7.- The observed spectra of I16293B. The arrangement of frequency and windows is the same as Figure 6. The column $\mathrm{v}_{L S R}$ ranges are -54.4 to $30,-42.7$ to $41.6,-24.8$ to 56.6 and -36.8 to 44.6 $\mathrm{km} \mathrm{s}^{-1}$, respectively. 


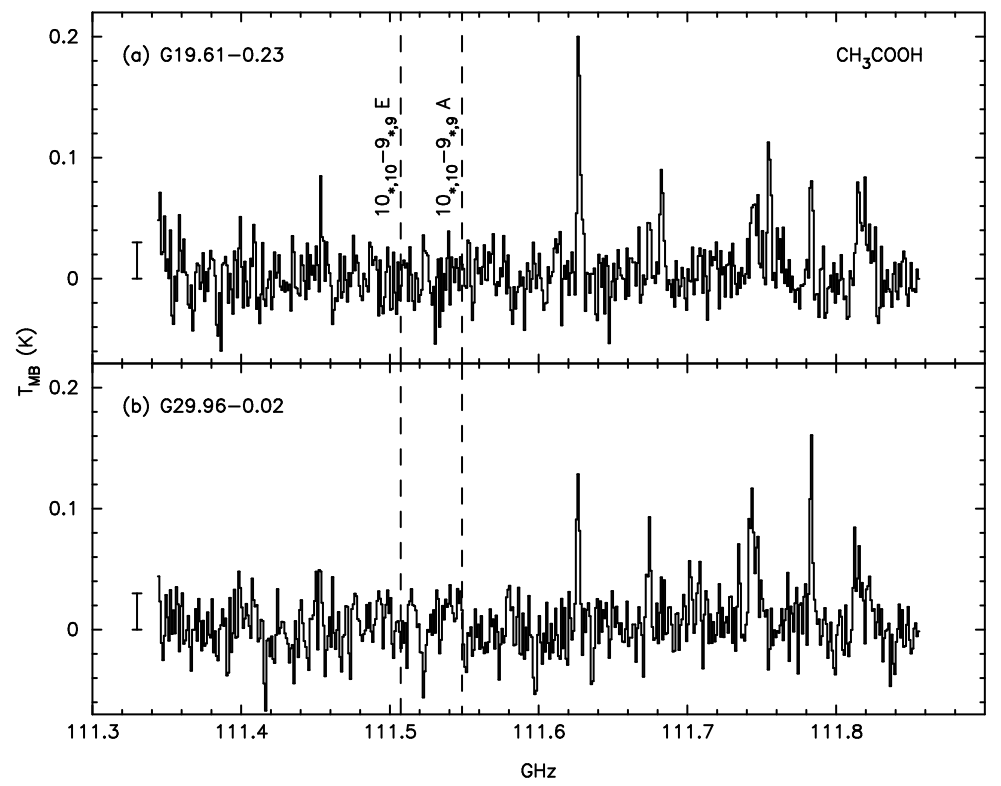

Fig. 8.- IRAM 30-m observations toward G19.61-0.23 and G29.96-0.02 (Fontani et al. 2007). The acetic acid lines are covered in this window at 111.50727 and $111.54853 \mathrm{GHz}$. 